Bundesgesundheitsbl 2013 $\cdot 56: 1706-1728$

DOI 10.1007/s00103-013-1863-6

๑) Springer-Verlag Berlin Heidelberg 2013

Bekanntmachung des Robert Koch-Institutes

\title{
Liste der vom Robert Koch-Institut geprüften und anerkannten Desinfektionsmittel und -verfahren
}

\author{
Stand: 31. August 2013
}

Die Liste gibt den derzeitigen Stand abschließend wieder; sie tritt an die Stelle der früheren, zuletzt im Bundesgesundheitsblatt 10 (2007):1335-1356 veröffentlichten Liste.

\section{Vorbemerkung}

Bei der A nwendung der nachstehend aufgeführten Mittel und Verfahren ist deren mikrobiologisches Wirkungsspektrum zu berücksichtigen. Die Wirkungsbereiche sind durch Buchstaben gekennzeichnet; es bedeuten:

\begin{tabular}{|l}
\hline Inhaltsübersicht \\
\hline Vorbemerkung \\
Mittel und Verfahren \\
1 Thermische Verfahren \\
1.1 Verbrennen \\
1.2 Kochen \\
1.3 Dampfdesinfektionsverfahren \\
2 Chemische Mittel und Verfahren \\
2.1 Instrumentendesinfektion \\
2.2 Wäschedesinfektion, Flächendesinfek- \\
tion (Wischdesinfektion), Desinfektion \\
von Ausscheidungen \\
2.3 Hygienische Händedesinfektion \\
3 Besondere Verfahren \\
3.1 Wäschedesinfektion in Waschmaschi- \\
nen \\
3.2 Instrumentendesinfektion in Reini- \\
gungs- und Desinfektionsgeräten \\
3.3 Raumdesinfektion \\
3.4 Desinfektion von Abfällen \\
3.5 Sonderverfahren zur Behandlung von \\
HEPA-Filtern in Sicherheitswerkbän- \\
ken (Klasse 2) \\
Anschriften der Hersteller bzw. Lieferfirmen \\
Anhang: Desinfektion spezieller Oberflächen \\
\hline
\end{tabular}

A: zur Abtötung von vegetativen Bakterien einschließlich Mykobakterien sowie von Pilzen einschließlich Pilzsporen geeignet,

B: zur Inaktivierung von Viren geeignet,

C: zur Abtötung von Sporen des Erregers des Milzbrandes geeignet,

D: zur Abtötung von Sporen der Erreger von Gasödem und Wundstarrkrampf geeignet; (zur Abtötung dieser Sporen müssen Sterilisationsverfahren unter Berücksichtigung der einschlägigen Normen angewendet werden).

Bezüglich der Wirksamkeit von Desinfektionsmaßnahmen gegen den Erreger der Creutzfeldt-Jakob-Erkrankung einschließlich seiner neuen Variante wird auf die Verlautbarungen im Bundesgesundheitsblatt 39 (1996):282-283, 41 (1998):279-285, 45 (2002):376-394, 47 (2004):36-40 und 55 (2012):1244-1310 verwiesen.

Angaben zu Art und Umfang von Desinfektionsmaßnahmen bei bestimmten Infektionskrankheiten sind in weiteren
Veröffentlichungen des Robert Koch-Instituts enthalten (http://www.rki.de/DE/ Content/Infekt/Krankenhaushygiene/ ThemenAZ/ThemenAZ_node.html).

Informationen zur chemischen Desinfektion der Sporen des Erregers des Milzbrandes enthält die Empfehlung des Robert Koch-Institutes zur „Vorgehensweise bei Verdacht auf Kontamination mit gefährlichen Erregern“ (http://www.rki.de/ DE/Content/Infekt/Biosicherheit/Agenzien/Vorgehensweise_Kontamination. pdf?_blob=publicationFile).

Bei der Anwendung der Desinfektionsmittel und -verfahren ist auch ihre Verträglichkeit mit den zu desinfizierenden Objekten zu beachten.

\section{Mittel und Verfahren}

\section{Thermische Verfahren}

\subsection{Verbrennen}

(Wirkungsbereich: ABCD)

\subsection{Kochen mit Wasser}

Desinfektionstemperatur: $100^{\circ} \mathrm{C}$ Einwirkungszeit: mind. 3 min (Wirkungsbereich: $A B$ ) mind. 15 min (Wirkungsbereich: $A B C$ )

\subsection{Dampfdesinfektionsverfahren}

Die hier aufgeführten Dampfdesinfektionsverfahren dienen zur Desinfektion von kontaminierten Objekten, die bei Desinfektionstemperaturen bis $105^{\circ} \mathrm{C}$ beständig sind. Außerdem muss sichergestellt sein, dass die Luft aus dem Gut verdrängt werden kann. Die Einwirkungszeit rechnet von dem Zeitpunkt an, zu 
dem alle Teile des Gutes gesättigtem Wasserdampf ausgesetzt sind und die Desinfektionstemperatur angenommen haben. Die Desinfektion poröser Güter (z. B. Bettenausstattungen, Matratzen) erfordert fraktionierte Vakuumverfahren. Die hier beschriebenen Verfahren sind für Abfälle, die gemäß IfSG desinfiziert werden müssen, nur unter Einhaltung der unter 3.4.3.1 bzw. 3.4.3.3.1 aufgeführten Anforderungen geeignet (Desinfektion von $\mathrm{Ab}$ fällen, s. 3.4).

Das bei der Durchführung der Verfahren anfallende Abwasser und die Abluft sind so nachzubehandeln, dass von ihnen keine Gefahren ausgehen können. Es sind die Anforderungen gemäß DIN 58 949, Teil 2 zu beachten. Die Desinfektionsanlagen sind entsprechend der Bedienungsanweisung $\mathrm{zu}$ beladen und $\mathrm{zu}$ betreiben, sie sind regelmäßig zu warten und auf Funktionstüchtigkeit zu prüfen. Auf DIN 58 949, Teil 3 wird diesbezüglich hingewiesen.

\subsubsection{Dampf-Strömungsverfahren}

Desinfektion in Apparaten mit gesättig tem Wasserdampf von mindestens $100^{\circ} \mathrm{C}$ Einwirkungszeit: mind. 5 min (Wirkungsbereich: $A B$ ) mind. 15 min (Wirkungsbereich: ABC)

\subsubsection{Fraktionierte}

\section{Vakuumverfahren (VDV-Verfahren)}

Die Verfahren sind gekennzeichnet durch:

1. Entfernung der Luft aus Kammer und Desinfektionsgut durch mehrmaliges Evakuieren im Wechsel mit Einströmen von Sattdampf,

2. Desinfektion mit Sattdampf,

3. Trocknen des Desinfektionsgutes durch Evakuieren.

Zur Durchführung dieser Verfahren ist Dampf erforderlich, der weitgehend frei von Luft- bzw. Fremdgasen ist (vgl. DIN EN 285). Die Desinfektionskammer muss vakuumdicht sein. Die vorgeschriebenen absoluten Drücke sind während der Vakuumphasen mit einer maximalen $\mathrm{Ab}$ weichung von +10 mbar und während der Zwischendampfstöße mit einer maximalen Abweichung von -10 mbar einzuhalten.

\subsubsection{System Belimed}

\section{Betriebsdaten}

a) $75^{\circ} \mathrm{C}$-Programm

Luftentfernung

Anzahl der Evakuierungsphasen: 3

In den Evakuierungsphasen zu erreichender Druck:

1. Phase: $12 \min \leq 25$ mbar

Folgende Phasen: $\leq 130$ mbar

Bei den Zwischendampfstößen zu erreichender Druck: $\geq 400$ mbar

\section{Desinfektion}

Dampftemperatur: $75^{\circ} \mathrm{C}$

Einwirkungszeit:

20 min (Wirkungsbereich: AB, außer Virushepatitis)

b) $105^{\circ} \mathrm{C}$-Programm

Luftentfernung

Anzahl der Evakuierungsphasen: 3

In den Evakuierungsphasen zu erreichender Druck:

1. Phase: 12 min $\leq 25$ mbar

Folgende Phasen: $\leq 400$ mbar

Bei den Zwischendampfstößen zu erreichender Druck: $\geq 1250$ mbar

Desinfektion

Dampftemperatur: $105^{\circ} \mathrm{C}$

Einwirkungszeit:

1 min (Wirkungsbereich: $\mathrm{AB}$ )

5 min (Wirkungsbereich: $A B C$ )

Geprüfte und anerkannte Apparatetypen: DV 650

\subsubsection{System Belimed Sauter}

\section{Betriebsdaten}

\section{Luftentfernung}

Anzahl der Evakuierungsphasen: 6

In den Evakuierungsphasen zu erreichender Druck: $\leq 70$ mbar

Bei den Zwischendampfstößen zu erreichender Druck: $\geq 300$ mbar

\section{Desinfektion}

a) $75^{\circ} \mathrm{C}$-Programm

Dampftemperatur: $75^{\circ} \mathrm{C}$

Einwirkungszeit:

20 min (Wirkungsbereich: AB, außer

Virushepatitis) b) $105^{\circ} \mathrm{C}$-Programm

Dampftemperatur: $105^{\circ} \mathrm{C}$

Einwirkungszeit:

1 min (Wirkungsbereich: AB)

5 min (Wirkungsbereich: ABC)

Geprüfter und anerkannter Apparatetyp:

DDA 7

\subsubsection{System Miele}

\section{Betriebsdaten}

a) $75^{\circ} \mathrm{C}$-Programm

Luftentfernung

Anzahl der Evakuierungsphasen: 4

In den Evakuierungsphasen zu erreichender Druck:

1. Phase: $\leq 50$ mbar

Folgende Phasen: $\leq 120$ mbar

Während der 1. Evakuierungsphase wird nach Erreichen von 50 mbar Dampf in die Kammer bis zu einem Druck von 400 mbar eingegeben und evakuiert bis auf 50 mbar.

Bei den Zwischendampfstößen zu erreichender Druck: $\geq 400$ mbar

Desinfektion

Dampftemperatur: $75^{\circ} \mathrm{C}$

Einwirkungszeit:

20 min (Wirkungsbereich: AB, außer

Virushepatitis)

b) $105^{\circ} \mathrm{C}$-Programm

Luftentfernung

Anzahl der Evakuierungsphasen: 4

In den Evakuierungsphasen zu erreichender Druck:

1. Phase: $\leq 50$ mbar

Folgende Phasen: $\leq 300$ mbar

Während der 1. Evakuierungsphase wird nach Erreichen von 50 mbar Dampf in die Kammer bis zu einem Druck von 400 mbar eingegeben und evakuiert bis auf 50 mbar.

Bei den Zwischendampfstößen zu erreichender Druck: $\geq 1000$ mbar

Desinfektion

Dampftemperatur: $105^{\circ} \mathrm{C}$

Einwirkungszeit:

1 min (Wirkungsbereich: AB)

5 min (Wirkungsbereich: $A B C$ ) 
Geprüfte und anerkannte Apparatetypen: DGD 8801, DGD 8802, DGD 8803, DGD 8804, DGD 8805

\subsubsection{System MMM}

\section{Betriebsdaten}

\section{Luftentfernung}

Anzahl der Evakuierungsphasen: 5

In den Evakuierungsphasen zu erreichender Druck: $\leq 80$ mbar

Bei den Zwischendampfstößen zu erreichender Druck: $\geq 400$ mbar

\section{Desinfektion}

a) $75^{\circ} \mathrm{C}$-Programm

Dampftemperatur: $75^{\circ} \mathrm{C}$

Einwirkungszeit:

20 min (Wirkungsbereich: AB, außer

Virushepatitis)

\section{b) $105^{\circ} \mathrm{C}$-Programm}

Dampftemperatur: $105^{\circ} \mathrm{C}$

Einwirkungszeit:

1 min (Wirkungsbereich: $A B$ )

5 min (Wirkungsbereich: ABC)

Geprüfte und anerkannte Apparatetypen: DES 1500/1501, DES 2000/2001, DES 3000/3001, DES 4000/4001, DES 6000/6001 sowie die baugleichen Typen Vacudes

\subsubsection{System Webeco}

\section{Betriebsdaten}

\section{Luftentfernung}

Anzahl der Evakuierungsphasen: 5

In den Evakuierungsphasen zu erreichender Druck: $\leq 80$ mbar

Bei den Zwischendampfstößen zu erreichender Druck: $\geq 400$ mbar

\section{Desinfektion}

\section{a) $75^{\circ} \mathrm{C}$-Programm}

Dampftemperatur: $75^{\circ} \mathrm{C}$

Einwirkungszeit:

20 min (Wirkungsbereich: AB, außer Virushepatitis)

\section{b) $105^{\circ} \mathrm{C}$-Programm}

Dampftemperatur: $105^{\circ} \mathrm{C}$

Einwirkungszeit:

7 min (Wirkungsbereich: ABC)
Geprüfte und anerkannte Apparatetypen: LD 210, LD 215, LD 220, LD 225, LD 230, LD 235, LD 237, LD 240, LD 250 sowie die entsprechenden Typen der Reihe LDH

In früheren Ausgaben der Liste aufgeführte und vorstehend nicht mehr verzeichnete Apparatetypen können weiterhin betrieben werden, sofern die vorgeschriebenen Betriebsdaten und die unter Ziffer 1.3 aufgeführten Bedingungen eingehalten werden sowie die regelmäßige Prüfung auf Funktionstüchtigkeit sichergestellt ist.

\section{Chemische Mittel und Verfahren}

Die Gebrauchsverdünnungen der chemischen Mittel sind mit reinem Wasser herzustellen; ein Zusatz von Reinigungsmitteln oder Ähnlichem ist nicht zulässig.

Werden zur Herstellung der Gebrauchsverdünnungen Desinfektionsmitteldosiergeräte verwendet, so sollen diese gemäß den von der Bundesanstalt für Materialforschung und -prüfung (BAM) und dem Bundesgesundheitsamt herausgegebenen Richtlinien [Bundesgesundheitsblatt 21 (1978):115-119 und 29 (1986):167168] bzw. seit 2004 gemäß der gemein- samen Empfehlung von BAM, RKI und Kommission für Krankenhaushygiene und Infektionsprävention „Anforderungen an Gestaltung, Eigenschaften und Betrieb von dezentralen Desinfektionsmittel-Dosiergeräten " [Bundesgesundheitsblatt 47 (2004):67-72] geprüft worden sein und die jeweiligen Anforderungen erfüllen. Die bei der Prüfung durch die BAM erteilten Auflagen und Hinweise zum Betrieb sind zu beachten.

\subsection{Instrumentendesinfektion}

\section{Siehe $\bullet$ Tabelle 1.}

Für die Aufbereitung von Medizinprodukten sind nur Desinfektionsmittel zulässig, deren Wirksamkeit für den Wirkungsbereich $\mathrm{AB}$ nachgewiesen ist. Auf die Empfehlung der Kommission für Krankenhaushygiene und Infektionsprävention beim RKI und des Bundesinstitutes für Arzneimittel und Medizinprodukte (Anforderungen an die Hygiene bei der Aufbereitung von Medizinprodukten Bundesgesundheitsblatt 55 (2012):12441310) wird verwiesen.

\section{Tab. 1 Instrumentendesinfektion}

\begin{tabular}{|c|c|c|c|c|c|}
\hline Wirkstoff & Name & $\begin{array}{l}\text { Konzentra- } \\
\text { tion GV in \% }\end{array}$ & EWZ in $\min$ & $\begin{array}{l}\text { Wirkungs- } \\
\text { bereich }\end{array}$ & $\begin{array}{l}\text { Hersteller bzw. } \\
\text { Lieferfirma }\end{array}$ \\
\hline \multirow{9}{*}{$\begin{array}{l}\text { Formaldehyd } \\
\text { und/oder } \\
\text { sonstige } \\
\text { Aldehyde } \\
\text { bzw. Derivate }\end{array}$} & Descoton forte & 3 & 120 & A & Dr. Schumacher \\
\hline & & 5 & 60 & A & \\
\hline & $\begin{array}{l}\text { Formaldehyd- } \\
\text { Lösung DAB } 10 \\
\text { (Formalin) }\end{array}$ & 6 & 60 & $A B$ & \\
\hline & Korsolex basic & 1 & 240 & A & Bode Chemie \\
\hline & & 2 & 120 & A & \\
\hline & & 3 & 60 & $A B$ & \\
\hline & & 4 & 30 & $A$ & \\
\hline & Lysetol V & 4 & 60 & $A B$ & Schülke \& Mayr \\
\hline & Sekusept forte & 7,5 & 60 & A & Ecolab \\
\hline \multirow{3}{*}{$\begin{array}{l}\text { Perverbin- } \\
\text { dungen }\end{array}$} & Peressigsäure $^{\mathrm{a}}$ & 0,35 & 60 & $A B$ & \\
\hline & \multirow[t]{2}{*}{ Sekusept aktiv } & 2 & 60 & A & Ecolab \\
\hline & & 7 & 60 & B & \\
\hline Phenole & $\begin{array}{l}\text { m-Kresol- } \\
\text { seifenlösung } \\
\text { DAB } 6\end{array}$ & 1,5 & 60 & A & \\
\hline $\begin{array}{l}\text { Sonstige } \\
\text { Wirkstoffe }\end{array}$ & Sekusept PLUS & 1,5 & 240 & A & Ecolab \\
\hline $\begin{array}{l}\text { aKonzentration } \\
\text { GV Gebrauchsv }\end{array}$ & $\begin{array}{l}\text { abe bezogen au } \\
\text { innung, EWZEin }\end{array}$ & $\begin{array}{l}\text { nen Wirkstoffg } \\
\text { kungszeit }\end{array}$ & & & haften beachten. \\
\hline
\end{tabular}




\subsection{Wäschedesinfektion, \\ Flächendesinfektion \\ (Wischdesinfektion), Desinfektion \\ von Ausscheidungen \\ Siehe $\bullet$ Tabelle 2 .}

\section{Tab. 2 Desinfektion von Wäsche, Flächen und Ausscheidungen}

\begin{tabular}{|c|c|c|c|c|c|c|c|c|c|c|c|c|c|}
\hline \multirow[t]{4}{*}{ Wirkstoff } & \multirow[t]{4}{*}{ Name } & \multirow{2}{*}{\multicolumn{2}{|c|}{$\begin{array}{l}\text { Wäschedes- } \\
\text { infektion } \\
\text { (Einlegever- } \\
\text { fahren) }\end{array}$}} & \multirow{2}{*}{\multicolumn{2}{|c|}{$\begin{array}{l}\text { Flächendes- } \\
\text { infektion } \\
\text { (Wischdesin- } \\
\text { fektion) }\end{array}$}} & \multicolumn{6}{|c|}{$\begin{array}{l}\text { Desinfektion von Ausscheidungen } 1 \text { Teil Aus- } \\
\text { wurf oder Stuhl + } 2 \text { Teile GV bzw. } 1 \text { Teil Harn } \\
+1 \text { Teil GV }\end{array}$} & \multirow[t]{4}{*}{$\begin{array}{l}\text { Wirkungs- } \\
\text { bereich }\end{array}$} & \multirow[t]{4}{*}{$\begin{array}{l}\text { Hersteller } \\
\text { bzw. } \\
\text { Lieferfirma }\end{array}$} \\
\hline & & & & & & \multicolumn{2}{|c|}{ Auswurf } & \multicolumn{2}{|c|}{ Stuhl } & \multicolumn{2}{|c|}{ Harn } & & \\
\hline & & GV & EWZ & GV & EWZ & GV & EWZ & GV & EWZ & GV & EWZ & & \\
\hline & & $\%$ & h & $\%$ & $\min$ & $\%$ & $\min$ & $\%$ & $\min$ & $\%$ & $\min$ & & \\
\hline Alkohole & Bacillol AF & & & Konz. & 15 & & & & & & & A & Bode Chemie \\
\hline Biguanide & Incidin Plus & & & 8 & 360 & & & & & & & $A$ & Ecolab \\
\hline \multirow{3}{*}{$\begin{array}{l}\text { Chlor, organ. } \\
\text { oder anorgan. } \\
\text { Substanzen mit } \\
\text { aktivem Chlor }\end{array}$} & $\begin{array}{l}\text { Chloramin-T } \\
\text { DAB } 9\end{array}$ & 1,5 & 12 & 2,5 & 120 & 5 & 240 & & & & & $A^{a} B$ & \\
\hline & Clorina & 1,5 & 12 & 2,5 & 120 & 5 & 240 & & & & & $A^{a} B$ & Lysoform \\
\hline & Trichlorol & 2 & 12 & 3 & 120 & 6 & 240 & & & & & $A^{a} B$ & Lysoform \\
\hline \multirow{19}{*}{$\begin{array}{l}\text { Formaldehyd } \\
\text { und/oder sons- } \\
\text { tige Aldehyde } \\
\text { bzw. Derivate }\end{array}$} & Aldasan 2000 & & & 4 & 240 & & & & & & & $A B$ & Lysoform \\
\hline & B 5 & & & 7 & 240 & & & & & & & $A B^{*}$ & orochemie \\
\hline & $\begin{array}{l}\text { Desifor } \\
\text { Protect }\end{array}$ & & & 7 & 240 & & & & & & & $A B^{*}$ & $\begin{array}{l}\text { Dr. Schnell } \\
\text { Chemie }\end{array}$ \\
\hline & $\begin{array}{l}\text { Desomed } \\
\text { Perfekt }\end{array}$ & & & 7 & 240 & & & & & & & $A B^{*}$ & Desomed \\
\hline & $\begin{array}{l}\text { ERVE NORO- } \\
\text { CID }\end{array}$ & & & 7 & 240 & & & & & & & $A B^{*}$ & $\begin{array}{l}\text { ERVE } \\
\text { Deutschland }\end{array}$ \\
\hline & $\begin{array}{l}\text { Formalde- } \\
\text { hyd-Lösung } \\
\text { DAB 10, } \\
\text { (Formalin) }\end{array}$ & 1,5 & 12 & 3 & 240 & & & & & & & $A B^{*}$ & \\
\hline & $\begin{array}{l}\text { Hygienic } \\
\text { VIRUZID }\end{array}$ & & & 7 & 240 & & & & & & & $A B^{*}$ & $\begin{array}{l}\text { Hagleitner } \\
\text { Hygiene }\end{array}$ \\
\hline & $\begin{array}{l}\text { Incidin } \\
\text { perfekt }\end{array}$ & 1 & 12 & 3 & 240 & & & & & & & $A B$ & Ecolab \\
\hline & $\begin{array}{l}\text { Kohrsolin } \\
\text { extra }\end{array}$ & & & 6 & 120 & & & & & & & $A B^{*}$ & Bode Chemie \\
\hline & Lysoform d & 4 & 12 & 5 & 360 & & & & & & & $A B$ & Lysoform \\
\hline & Lysoformin & 3 & 12 & 5 & 360 & & & & & & & $A B$ & Lysoform \\
\hline & $\begin{array}{l}\text { Lysoformin } \\
2000\end{array}$ & & & 4 & 360 & & & & & & & $A B$ & Lysoform \\
\hline & Melsept & 2 & 12 & 4 & 360 & & & & & & & $A B$ & B. Braun \\
\hline & Melsitt & 4 & 12 & 10 & 240 & & & & & & & $A B$ & B. Braun \\
\hline & Minutil & 2 & 12 & 6 & 240 & & & & & & & $A B$ & Ecolab \\
\hline & Nüscosept & & & 5 & 240 & & & & & & & $A B$ & $\begin{array}{l}\text { Dr. Nüsken } \\
\text { Chemie }\end{array}$ \\
\hline & Optisept & & & 7 & 240 & & & & & & & $A B^{*}$ & $\begin{array}{l}\text { Dr. Schuma- } \\
\text { cher }\end{array}$ \\
\hline & Pursept-FD & & & 7 & 240 & & & & & & & $A B^{*}$ & $\begin{array}{l}\text { Merz Hygiene } \\
\text { GmbH }\end{array}$ \\
\hline & Ultrasol F & 3 & 12 & 5 & 240 & & & & & & & $A B$ & $\begin{array}{l}\text { Dr. Schuma- } \\
\text { cher }\end{array}$ \\
\hline Lauge & Kalkmilch ${ }^{c}$ & & & & & & & 20 & 360 & & & $A^{C} B$ & \\
\hline
\end{tabular}




\begin{tabular}{|c|c|c|c|c|c|c|c|c|c|c|c|c|c|}
\hline \multirow[t]{4}{*}{ Wirkstoff } & \multirow[t]{4}{*}{ Name } & \multirow{2}{*}{\multicolumn{2}{|c|}{$\begin{array}{l}\text { Wäschedes- } \\
\text { infektion } \\
\text { (Einlegever- } \\
\text { fahren) }\end{array}$}} & \multirow{2}{*}{\multicolumn{2}{|c|}{$\begin{array}{l}\text { Flächendes- } \\
\text { infektion } \\
\text { (Wischdesin- } \\
\text { fektion) }\end{array}$}} & \multicolumn{6}{|c|}{$\begin{array}{l}\text { Desinfektion von Ausscheidungen } 1 \text { Teil Aus- } \\
\text { wurf oder Stuhl + } 2 \text { Teile Gebr.-Verd. bzw. } 1 \text { Teil } \\
\text { Harn +1 Teil Gebr.-Verd. }\end{array}$} & \multirow[t]{4}{*}{$\begin{array}{l}\text { Wirkungs- } \\
\text { bereich }\end{array}$} & \multirow[t]{4}{*}{$\begin{array}{l}\text { Hersteller } \\
\text { bzw. } \\
\text { Lieferfirma }\end{array}$} \\
\hline & & & & & & \multicolumn{2}{|c|}{ Auswurf } & \multicolumn{2}{|c|}{ Stuhl } & \multicolumn{2}{|c|}{ Harn } & & \\
\hline & & GV & EWZ & GV & EWZ & GV & EWZ & GV & EWZ & GV & EWZ & & \\
\hline & & $\%$ & h & $\%$ & $\min$ & $\%$ & $\min$ & $\%$ & $\min$ & $\%$ & $\min$ & & \\
\hline \multirow[t]{14}{*}{$\begin{array}{l}\text { Perverbindun- } \\
\text { gen }\end{array}$} & $\begin{array}{l}\text { Apesin } \\
\text { AP100 }\end{array}$ & & & 4 & 240 & & & & & & & $A B$ & $\begin{array}{l}\text { Tana PROFES } \\
\text { SIONAL }\end{array}$ \\
\hline & $\begin{array}{l}\text { Dismozon } \\
\text { plus }^{\text {b }}\end{array}$ & & & 3,6 & 240 & & & & & & & $A B^{*}$ & Bode Chemie \\
\hline & $\begin{array}{l}\text { Dismozon } \\
\text { plus }^{b}\end{array}$ & & & 3,6 & 15 & & & & & & & $B^{*}$ & Bode Chemie \\
\hline & $\begin{array}{l}\text { Dismozon } \\
\text { pur }\end{array}$ & & & 4 & 60 & & & & & & & $A B$ & Bode Chemie \\
\hline & $\begin{array}{l}\text { Incidin } \\
\text { active }^{b}\end{array}$ & & & 3 & 60 & & & & & & & $A B^{*}$ & $\begin{array}{l}\text { Ecolab } \\
\text { Deutschland }\end{array}$ \\
\hline & $\begin{array}{l}\text { Incidin } \\
\text { active }^{\text {b }}\end{array}$ & & & 2 & 60 & & & & & & & $\mathrm{~B}^{*}$ & $\begin{array}{l}\text { Ecolab } \\
\text { Deutschland }\end{array}$ \\
\hline & Perform ${ }^{b}$ & & & 3 & 240 & & & & & & & $A B$ & $\begin{array}{l}\text { Schülke \& } \\
\text { Mayr }\end{array}$ \\
\hline & Terralin $P A A^{b}$ & & & 8 & 60 & & & & & & & $A B^{*}$ & $\begin{array}{l}\text { Schülke \& } \\
\text { Mayr }\end{array}$ \\
\hline & Terralin $P A A^{b}$ & & & 7 & 60 & & & & & & & $B^{*}$ & $\begin{array}{l}\text { Schülke \& } \\
\text { Mayr }\end{array}$ \\
\hline & Wofasteril $^{b}$ & & & 2 & 60 & & & & & & & A & Kesla Pharma \\
\hline & Wofasteril $^{\mathrm{b}}$ & & & 2 & 240 & & & & & & & $A B$ & Kesla Pharma \\
\hline & $\begin{array}{l}\text { Wofasteril- } \\
\text { Kombiver- } \\
\text { fahren }\end{array}$ & & & & & & & & & & & & Kesla Pharma \\
\hline & $\begin{array}{l}\text { - Wofasteril } \\
\text { und }\end{array}$ & & & 2 & 60 & & & & & & & A & \\
\hline & Alcapur & & & 6 & & & & & & & & & \\
\hline \multirow{4}{*}{$\begin{array}{l}\text { Phenol oder } \\
\text { Phenolderivate }\end{array}$} & Amocid & 1 & 12 & 5 & 360 & 5 & 240 & 5 & 360 & 5 & 120 & A & Lysoform \\
\hline & Helipur & & & 6 & 240 & 6 & 240 & 6 & 360 & 6 & 120 & A & B. Braun \\
\hline & $\begin{array}{l}\text { m-Kresol- } \\
\text { seifenlösung } \\
\text { DAB 6 }\end{array}$ & 1 & 12 & 5 & 240 & & & & & & & A & \\
\hline & Phenol & 1 & 12 & 3 & 120 & & & & & & & $A$ & \\
\hline \multicolumn{14}{|c|}{ 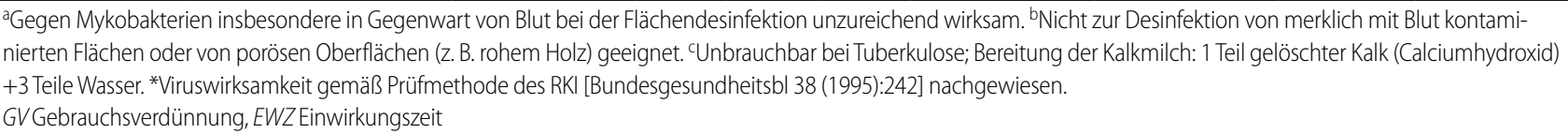 } \\
\hline
\end{tabular}




\subsection{Hygienische Händedesinfektion} Siehe - Tabelle 3.

Die Hände werden mit der Lösung eingerieben und während der vorgeschriebenen Einwirkungszeit feucht gehal- ten. Die in der Tabelle aufgeführten Zeiten sind Mindestwerte. Bei massiver bzw. sichtbarer Kontamination und bei Kontamination mit Tuberkulose-Bakterien ist die Desinfektion zweimal durchzuführen.
Dem auf den Händen verteilten Desinfektionsmittel darf Wasser erst nach Ablauf der für die Desinfektion vorgesehenen Einwirkungszeit zugesetzt werden.

Tab. 3 Hygienische Händedesinfektion

\begin{tabular}{|c|c|c|c|c|c|}
\hline \multirow[t]{2}{*}{ Wirkstoff } & \multirow[t]{2}{*}{ Name } & \multicolumn{3}{|c|}{ Wirkungsbereich/Einwirkzeiten in min } & \multirow[t]{2}{*}{ Hersteller bzw. Lieferfirma } \\
\hline & & A & Begrenzt viruzid & $B$ (viruzid) & \\
\hline \multirow[t]{46}{*}{ Alkohole $^{\mathrm{a}}$} & AHD 2000 & $1 / 2$ & $1 / 2$ & & Lysoform \\
\hline & Aktivin DHH & $1 / 2$ & & & Fritz Osk. Michallik \\
\hline & Aseptoman & $1 / 2$ & & & Dr. Schumacher \\
\hline & Aseptoman Viral & $1 / 2$ & & $1^{\#, c}$ & Dr. Schumacher \\
\hline & Aseptopur & $1 / 2$ & & & Desomed \\
\hline & $\mathrm{C} 20$ & $1 / 2$ & $1 / 2$ & & orochemie \\
\hline & Cimo Skin & $1 / 2$ & & & Dr. Schnell Chemie \\
\hline & Descoderm & $1 / 2$ & & & Dr. Schumacher \\
\hline & Desderman pure & $1 / 2$ & & & Schülke \& Mayr \\
\hline & ERVESEPT & $1 / 2$ & & & ERVE Deutschland \\
\hline & Ethanol DAB 10, 80 Vol.\% & $1 / 2$ & & & \\
\hline & FINK-Antisept HD med & $1 / 2$ & & & Fink Tec GmbH \\
\hline & HD 410 & $1 / 2$ & $1 / 2$ & & orochemie \\
\hline & Hospisept & $1 / 2$ & $1 / 2$ & & Lysoform \\
\hline & Isopropanol 70 Vol.\% & $1 / 2$ & & & \\
\hline & Kaniderm & $1 / 2$ & & & Kaniedenta Dental \\
\hline & Kentoman & $1 / 2$ & & & NORDENTA \\
\hline & Kodan-Tinktur forte & $1 / 2$ & & & Schülke \& Mayr \\
\hline & Manorapid Synergy & $1 / 2$ & $1 / 2$ & $1^{\#, c}$ & Antiseptica \\
\hline & mobilomed Skinsoft & $1 / 2$ & & & mobiloclean \\
\hline & Mucasept-A & $1 / 2$ & & & Merz Hygiene GmbH \\
\hline & Novaderm & $1 / 2$ & & & Novapraxishygiene \\
\hline & Nüscoman & $1 / 2$ & & & Dr. Nüsken Chemie \\
\hline & OP-Sept & $1 / 2$ & & & Laboratorium Dr. Deppe \\
\hline & Poly-Alcohol Hände-Antisepticum & $1 / 2$ & $1 / 2$ & & Antiseptica \\
\hline & Poly-Alcohol Haut farblos Antisepticum & $1 / 2$ & & & Antiseptica \\
\hline & Promanum pure & $1 / 2$ & $1 / 2$ & & B. Braun \\
\hline & n-Propanol 60 Vol.\% & $1 / 2$ & & & \\
\hline & SARAYA Haut- \& Händedesinfektion & $1 / 2$ & & & SARAYA Europe \\
\hline & Sensiva Händedesinfektion & $1 / 2$ & & & Schülke \& Mayr \\
\hline & Septoderm Hände & $1 / 2$ & & & Dr. Schumacher \\
\hline & Skinman clear & $1 / 2$ & $1 / 2$ & & Ecolab \\
\hline & Skinman complete & $1 / 2$ & & & Ecolab \\
\hline & Skinman soft & $1 / 2$ & $1 / 2$ & & Ecolab \\
\hline & Skinsept F & $1 / 2$ & & & Ecolab \\
\hline & Softa-Man & $1 / 2$ & $1 / 2$ & & B. Braun \\
\hline & Softa-Man acute & $1 / 2$ & & $1^{\#, c}$ & B. Braun \\
\hline & Spitacid & $1 / 2$ & $1 / 2$ & & Ecolab \\
\hline & Sterillium & $1 / 2$ & $1 / 2$ & & Bode Chemie \\
\hline & Sterillium classic pure & $1 / 2$ & $1 / 2$ & & Bode Chemie \\
\hline & Sterillium med & $1 / 2$ & $1 / 2$ & & Bode Chemie \\
\hline & Sterillium Virugard & $1 / 2$ & $1 / 2$ & $2^{\#, c}$ & Bode Chemie \\
\hline & Steritan & $1 / 2$ & $1 / 2$ & & Sanitätsamt der Bundeswehr \\
\hline & Suprades HD & $1 / 2$ & & & Anti-Germ Deutschland GmbH \\
\hline & triformin medDIS & $1 / 2$ & & & Dr. Weigert \\
\hline & VIRUSEPT Manorapid Synergy & $1 / 2$ & & $1^{\#, c}$ & Merz Hygiene GmbH \\
\hline
\end{tabular}




\begin{tabular}{|c|c|c|c|c|c|}
\hline \multirow[t]{2}{*}{ Wirkstoff } & \multirow[t]{2}{*}{ Name } & \multicolumn{3}{|c|}{ Wirkungsbereich/Einwirkzeiten in min } & \multirow[t]{2}{*}{ Hersteller bzw. Lieferfirma } \\
\hline & & A & Begrenzt viruzid & $B$ (viruzid) & \\
\hline \multirow[t]{5}{*}{ Halogene } & Braunol & 1 & & & B. Braun \\
\hline & Chloramin T (DAB 9) 1\% & $2^{b}$ & & $2^{\#, c}$ & \\
\hline & Chloramin T (DAB 9) 2\% & $1^{\mathrm{b}}$ & $1^{\mathrm{c}}$ & $1^{\#, c}$ & \\
\hline & Chloramin T-Lysoform 1\% & $2^{b}$ & & $2^{\#, c}$ & Lysoform \\
\hline & Chloramin T-Lysoform 2\% & $1^{\mathrm{b}}$ & $1^{c}$ & $1^{\#, c}$ & Lysoform \\
\hline \multirow{2}{*}{$\begin{array}{l}\text { Sonstige } \\
\text { Wirkstoffe }\end{array}$} & Primasept Med & 1 & & & Schülke \& Mayr \\
\hline & Wofasteril 0,5\% & 1 & 1 & & Kesla Pharma \\
\hline \multicolumn{6}{|c|}{$\begin{array}{l}\text { 'Die Einordnung der Präparate in diese Gruppe besagt nicht, dass die Mittel ausschließlich Alkohole als Wirkstoffe enthalten. Auskunft über weitere Wirkstoffe gibt die } \\
\text { Deklaration des Herstellers. }{ }^{\circ} \text { Gegen Mykobakterien und Pilze unzureichend wirksam. CUnzureichend wirksam bei sichtbaren Verschmutzungen. \#Viruzid wirksam gemäß } \\
\text { Stellungnahme des Arbeitskreises Viruzidie. Bundesgesundheitsbl (2004) 47:62-66 (http://www.rki.de/DE/Content/Infekt/Krankenhaushygiene/Desinfektionsmittel/Viruzid } \\
\text { pdf?_blob=publicationFile). }\end{array}$} \\
\hline
\end{tabular}

\section{Besondere Verfahren}

Die Apparate sind entsprechend der Bedienungsanweisung zu betreiben, regelmäßig zu warten und auf Funktionstüchtigkeit zu prüfen.

\subsection{Wäschedesinfektion in Waschmaschinen}

Die Maschinen müssen gewährleisten, dass die für das jeweilige Verfahren vorgeschriebene Konzentration des Desinfektions- und des Waschmittels, das Flottenverhältnis und die Temperatur während der Einwirkungszeit eingehalten werden. Die für das Flottenverhältnis angegebenen Daten sind Mindestwerte. Es ist zulässig, größere Flotten anzuwenden.

(Flotte $=$ Flüssigkeitsmenge, mit der das Reinigungsgut während einer Arbeitsphase behandelt wird. Flottenverhältnis = Verhältnis der Gewichtsmengen von Reinigungsgut und Flotte.)

Am Ende der Desinfektionsphase müssen Desinfektionsgut, Flotte und der Innenraum der Maschine, der mit der kontaminierten Wäsche und der Flotte in Berührung kam, desinfiziert sein. Vor Beendigung der Desinfektionsphase darf keine Flotte aus der Maschine abfließen. Die Abluft ist so abzuführen bzw. nachzubehandeln, dass von ihr keine Gefahren ausgehen können.

Nach dem derzeitigen Stand der Technik können diese Forderungen von folgenden Waschmaschinen erfüllt werden:

1. diskontinuierlich arbeitende Trommelwaschmaschinen (Haushaltswaschmaschinen sind in der Regel nicht geeignet)
2. kontinuierlich arbeitende Waschmaschinen soweit sie nachführend aufgeführt sind.

Die bei der Eintragung vom Robert KochInstitut erteilten Auflagen, insbesondere hinsichtlich der Taktzeiten sind zu beachten.

a) Waschstraße Senking P 50/P 36
Hersteller: Jensen GmbH

b) Waschstraße Senking P 25

Hersteller: Jensen $\mathrm{GmbH}$

In Sondereinheiten für hochkontagiöse Krankheiten empfiehlt sich die Verwendung von Einmalwäsche. Ist dies nicht möglich, muss die Wäsche in der Sondereinheit thermisch, notfalls chemisch desinfiziert werden.

\subsubsection{Thermische Desinfektionswaschverfahren}

Die Konzentration der Waschmittel sollte den Empfehlungen der Hersteller entsprechen. Die waschtechnische Eignung der Waschmittel sollte durch Gutachten (textiltechnisches Gutachten) belegt sein.

a) Desinfektionstemperatur: $85^{\circ} \mathrm{C}$

Einwirkungszeit: $15 \mathrm{~min}$

b) Desinfektionstemperatur: $90^{\circ} \mathrm{C}$

Einwirkungszeit: $10 \mathrm{~min}$

Flottenverhältnis: 1:4 bis 1:5

Wirkungsbereich: AB

\subsubsection{Chemothermische Desinfektionswaschverfahren}

Die bei der Eintragung des jeweiligen Verfahrens im Bescheid aufgeführten Angaben zum Verfahrensablauf sind exakt einzuhalten, um seine Wirksamkeit zu gewährleisten.

Die Verfahren in $\bullet$ Tabelle 4 sind nicht für merklich mit Blut verschmutzte Wäsche geeignet. Die Verfahren in $\bullet$ Tabelle 5 sind nicht für stark verschmutzte und auch nicht für merklich mit Blut verschmutzte Wäsche geeignet. 
Tab. 4 Verfahren mit Perverbindungen als Wirkstoff

\begin{tabular}{|c|c|c|c|c|c|c|c|}
\hline \multirow[t]{2}{*}{ Name } & \multicolumn{2}{|c|}{ Konzentration (g auf 1 I Flotte) } & \multirow{2}{*}{$\begin{array}{l}\text { Desinfek- } \\
\text { tionstempe- } \\
\text { ratur in }{ }^{\circ} \mathrm{C}\end{array}$} & \multirow{2}{*}{$\begin{array}{l}\text { Einwirkungs- } \\
\text { zeit in min }\end{array}$} & \multirow{2}{*}{$\begin{array}{l}\text { Flotten- } \\
\text { verhältnis }\end{array}$} & \multirow{2}{*}{$\begin{array}{l}\text { Wirkungs- } \\
\text { bereich }\end{array}$} & \multirow{2}{*}{$\begin{array}{l}\text { Hersteller } \\
\text { bzw. } \\
\text { Lieferfirma }\end{array}$} \\
\hline & Waschmittel & $\begin{array}{l}\text { Desinfektions- } \\
\text { mittel }\end{array}$ & & & & & \\
\hline ARENAS-Verfahren & $5 \mathrm{ml}$ Arenas-wash & $\begin{array}{l}5 \mathrm{ml} \text { Arenas } \\
\text { oxydes }^{\mathrm{a}}\end{array}$ & 60 & 15 & $1: 4$ & A & $\begin{array}{l}\text { Johannes } \\
\text { Kiehl }\end{array}$ \\
\hline ARENAS-Verfahren & $\begin{array}{l}5 \mathrm{ml} \text { ARENAS- } \\
\text { wash und } 5 \mathrm{ml} \\
\text { ARENAS-excellent }\end{array}$ & $\begin{array}{l}5 \mathrm{ml} \text { ARENAS- } \\
\text { oxydes }^{\text {a }}\end{array}$ & 60 & 15 & $1: 4$ & $A B$ & $\begin{array}{l}\text { Johannes } \\
\text { Kiehl }\end{array}$ \\
\hline BA 52-Verfahren & $\begin{array}{l}2 \mathrm{~g} \text { Supertakt } \\
2000 \text { und } 2 \mathrm{~g} \\
\text { Waschverstärker }\end{array}$ & $2 \mathrm{gBA} 52^{\mathrm{a}}$ & 60 & 15 & $1: 5$ & $A B$ & $\begin{array}{l}\text { Schuster } \\
\text { Chemie }\end{array}$ \\
\hline BEICLEAN CONTROL-Verfahren & 1 ml Beipur PKS & $\begin{array}{l}1 \mathrm{ml} \text { Beibleach } \\
\text { WP } 35^{\mathrm{a}} \text { und } \\
0,8 \mathrm{ml} \mathrm{Beiclean}^{\text {Control }}{ }^{\mathrm{a}}\end{array}$ & 60 & 12 & $1: 5$ & $A B$ & CHT Beitlich \\
\hline $\begin{array}{l}\text { BEICLEAN HWA/BEIBLEACH WP35- } \\
\text { Verfahren }\end{array}$ & $\begin{array}{l}0,9 \mathrm{ml} \mathrm{BEIPUR} \\
\text { WAK }\end{array}$ & $\begin{array}{l}1,5 \mathrm{ml} \text { BEICLEAN } \\
\text { HWA und } 1 \mathrm{ml} \\
\text { BEIBLEACH } \\
\text { WP35 }\end{array}$ & 60 & 15 & $1: 5$ & $A B$ & CHT Beitlich \\
\hline Clax Personril-Verfahren & $4 \mathrm{~g} \mathrm{Clax} \mathrm{Diamond}$ & $\begin{array}{l}2 \mathrm{ml} \text { Clax Per- } \\
\text { sonril }\end{array}$ & 60 & 15 & $1: 5$ & A & $\begin{array}{l}\text { Diversey } \\
\text { Deutschland }\end{array}$ \\
\hline Peracid Asepsis-Verfahren & $\begin{array}{l}\text { 3-6 g Osmac K, } \\
\text { Prolong Powder } \\
\text { oder } 2-4 \mathrm{~g} \text { Os- } \\
\text { maflux }\end{array}$ & $\begin{array}{l}2 \mathrm{ml} \text { Peracid } \\
\text { Asepsis }^{\mathrm{a}}\end{array}$ & 60 & 15 & $1: 5$ & A & $\begin{array}{l}\text { Christeyns } \\
\text { GmbH }\end{array}$ \\
\hline Peracid Asepsis-Verfahren & $\begin{array}{l}\text { 3-6 g Kom- } \\
\text { bimax B, } 3 \mathrm{~g} \\
\text { Osmac K, Prolong } \\
\text { Powder oder } 2 \mathrm{~g} \\
\text { Osmaflux }\end{array}$ & $\begin{array}{l}2 \mathrm{ml} \text { Peracid } \\
\text { Asepsis }^{\mathrm{a}}\end{array}$ & 60 & 15 & $1: 5$ & $A B$ & $\begin{array}{l}\text { Christeyns } \\
\text { GmbH }\end{array}$ \\
\hline Peracid Asepsis-Verfahren & $\begin{array}{l}1,2-2,4 \mathrm{~g} \text { Majestic } \\
\text { green }\end{array}$ & $\begin{array}{l}2 \mathrm{ml} \text { Peracid } \\
\text { Asepsis }^{\mathrm{a}}\end{array}$ & 70 & 10 & $1: 4$ & $A B$ & $\begin{array}{l}\text { Christeyns } \\
\text { GmbH }\end{array}$ \\
\hline Peracid Asepsis-Verfahren & $\begin{array}{l}\text { 1,2-2,4 g Majestic } \\
\text { classic }\end{array}$ & $\begin{array}{l}2 \mathrm{ml} \text { Peracid } \\
\text { Asepsis }^{\mathrm{a}}\end{array}$ & 70 & 10 & $1: 5$ & $A B$ & $\begin{array}{l}\text { Christeyns } \\
\text { GmbH }\end{array}$ \\
\hline Ottalin PA conc-Verfahren & 1,2 g Trebon Si & $\begin{array}{l}\text { 0,8 g Ottalin PA } \\
\text { conc }^{\mathrm{a}}\end{array}$ & 60 & 10 & $1: 5$ & $A B$ & Kreussler \\
\hline Ottalin Peracet-Verfahren & $\begin{array}{l}\text { 2-4 } \mathrm{ml} \text { Derval Solo } \\
\text { oder } 2 \mathrm{~g} \text { Trebon } \mathrm{Si}\end{array}$ & $\begin{array}{l}2 \mathrm{ml} \mathrm{Ottalin} \\
\text { Peracet }^{\mathrm{a}}\end{array}$ & 60 & 10 & $1: 5$ & $A B$ & Kreussler \\
\hline Ottalin Peracet-Verfahren & $2 \mathrm{~g}$ Trebon Basis & $\begin{array}{l}2 \mathrm{ml}^{\text {Ottalin }} \\
\text { Peracet }^{\mathrm{a}}\end{array}$ & 65 & 12 & $1: 5$ & A & Kreussler \\
\hline OXYPLEX-Verfahren & $\begin{array}{l}3 \mathrm{~g} \text { ALIPLEX, OSET- } \\
\text { TA, UNIPLEX oder } \\
4 \mathrm{ml} \text { OLISSO }\end{array}$ & 2 ml OXYPLPEXa & 60 & 15 & $1: 5$ & $A B$ & $\begin{array}{l}\text { Burnus- } \\
\text { Hychem }\end{array}$ \\
\hline OXYPLEX plus Verfahren & 1 ml Liquisan B & $\begin{array}{l}1 \mathrm{ml} \text { OXYPLEX } \\
\text { plus }^{\mathrm{a}}\end{array}$ & 60 & 10 & $1: 5$ & $A B$ & $\begin{array}{l}\text { Burnus- } \\
\text { Hychem }\end{array}$ \\
\hline OXYPLEX plus Verfahren & $\begin{array}{l}3 \text { g ALIPLEX, OSET- } \\
\text { TA, UNIPLEX oder } \\
4 \text { ml OLISSO }\end{array}$ & $\begin{array}{l}0,7 \mathrm{ml} \text { OXYPLEX } \\
\text { plus }^{\mathrm{a}}\end{array}$ & 70 & 10 & $1: 5$ & $A B$ & $\begin{array}{l}\text { Burnus- } \\
\text { Hychem }\end{array}$ \\
\hline Ozonit-Verfahren & $\begin{array}{l}2,5-5 \mathrm{~g} \text { Turbo } \\
\text { Power }\end{array}$ & $\begin{array}{l}2 \mathrm{ml} \mathrm{Ozonit}^{\mathrm{a}} \\
\text { oder } 1 \mathrm{ml} \text { Ozonit } \\
\text { super }^{\mathrm{a}}\end{array}$ & 60 & 15 & $1: 4$ & $A B$ & Ecolab \\
\hline Ozonit-Verfahren & $\begin{array}{l}3-6 \mathrm{~g} \text { Silex uni- } \\
\text { versal }\end{array}$ & $\begin{array}{l}1 \mathrm{ml} \text { Ozonit } \\
\text { super }^{\mathrm{a}}\end{array}$ & 60 & 15 & $1: 5$ & A & Ecolab \\
\hline Ozonit-Verfahren & $\begin{array}{l}\text { 3-6 g Almesin uni- } \\
\text { versal, Dermasil } \\
\text { perfekt oder Tri- } \\
\text { plex energy PF }\end{array}$ & $\begin{array}{l}2 \mathrm{ml} \mathrm{Ozonit}^{\mathrm{a}} \\
\text { oder } 1 \mathrm{ml} \text { Ozonit } \\
\text { super }^{\mathrm{a}}\end{array}$ & 60 & 15 & $1: 5$ & $A B$ & Ecolab \\
\hline
\end{tabular}




\begin{tabular}{|c|c|c|c|c|c|c|c|}
\hline \multirow[t]{2}{*}{ Name } & \multicolumn{2}{|c|}{ Konzentration (g auf 1 I Flotte) } & \multirow{2}{*}{$\begin{array}{l}\text { Desinfek- } \\
\text { tionstempe- } \\
\text { ratur in }{ }^{\circ} \mathrm{C}\end{array}$} & \multirow{2}{*}{$\begin{array}{l}\text { Einwirkungs- } \\
\text { zeit in min }\end{array}$} & \multirow{2}{*}{$\begin{array}{l}\text { Flotten- } \\
\text { verhältnis }\end{array}$} & \multirow{2}{*}{$\begin{array}{l}\text { Wirkungs- } \\
\text { bereich }\end{array}$} & \multirow{2}{*}{$\begin{array}{l}\text { Hersteller } \\
\text { bzw. } \\
\text { Lieferfirma }\end{array}$} \\
\hline & Waschmittel & $\begin{array}{l}\text { Desinfektions- } \\
\text { mittel }\end{array}$ & & & & & \\
\hline Ozonit-Verfahren & $\begin{array}{l}1,2 \mathrm{~g} \text { Compactat } \\
\text { Future }\end{array}$ & $\begin{array}{l}2 \mathrm{ml} \text { Ozonit } \\
\text { oder } 1 \mathrm{ml} \text { Ozonit } \\
\text { super }^{\mathrm{a}}\end{array}$ & 70 & 7 & $1: 4$ & $A B$ & Ecolab \\
\hline Ozonit-Verfahren & $\begin{array}{l}6 \mathrm{~g} \text { Triplex energy } \\
\text { oder 2,5-5 g Turbo } \\
\text { Power }\end{array}$ & $\begin{array}{l}2 \mathrm{ml} \text { Ozonita } \\
\text { oder } 1 \mathrm{ml} \text { Ozonit } \\
\text { super }^{\mathrm{a}}\end{array}$ & 70 & 10 & $1: 4$ & A & Ecolab \\
\hline Ozonit-Verfahren & $\begin{array}{l}\text { 1,5 g Dermasil } \\
\text { perfekt,1,5 g Silex } \\
\text { 3000, 3-6 g Silex } \\
\text { universal, } 3 \mathrm{~g} \text { Tri- } \\
\text { plex energy, 1,5 g } \\
\text { Triplex energy PF } \\
\text { oder 2,5 g Turbo } \\
\text { Power }\end{array}$ & $\begin{array}{l}2 \mathrm{ml} \mathrm{Ozonit}^{\mathrm{a}} \\
\text { oder } 1 \mathrm{ml} \text { Ozonit } \\
\text { super }^{\mathrm{a}}\end{array}$ & 70 & 10 & $1: 4$ & $A B$ & Ecolab \\
\hline Ozonit-Verfahren & $\begin{array}{l}\text { 1,5-6 g Membrex, } \\
\text { Membrex color } \\
\text { oder } 0,6-1,4 \mathrm{~g} \\
\text { Turbo Plus und } \\
1-2,4 \mathrm{~g} \text { Turbo } \\
\text { break }\end{array}$ & $\begin{array}{l}2 \mathrm{ml} \text { Ozonit }{ }^{\mathrm{a}} \\
\text { oder } 1 \mathrm{ml} \text { Ozonit } \\
\text { super }^{\mathrm{a}}\end{array}$ & 70 & 10 & $1: 5$ & A & Ecolab \\
\hline Ozonit-Verfahren & $\begin{array}{l}\text { 1,5 g Membrex, } \\
\text { Membrex color, } \\
\text { 3-6 g Almesin uni- } \\
\text { versal, Dermasil } \\
\text { perfekt, } 1,5 \mathrm{ml} \\
\text { Triplex Emulsion, } \\
\text { 3-6 g Triplex ener- } \\
\text { gy PF oder 1,4 g } \\
\text { Turbo Plus und } \\
\text { 2,4 g Turbo break }\end{array}$ & $\begin{array}{l}2 \mathrm{ml} \mathrm{Ozonit}^{\mathrm{a}} \\
\text { oder } 1 \mathrm{ml} \text { Ozonit } \\
\text { super }\end{array}$ & 70 & 10 & $1: 5$ & $A B$ & Ecolab \\
\hline Ozonit Performance-Verfahren & $\begin{array}{l}0,8 \mathrm{ml} \text { Turbo plus } \\
\text { und } 0,5 \mathrm{ml} \text { Turbo } \\
\text { break }\end{array}$ & $\begin{array}{l}0,7 \text { ml Ozonit } \\
\text { Performance }^{a}\end{array}$ & 60 & 10 & $1: 5$ & $A B$ & Ecolab \\
\hline Ozonit Performance-Verfahren & $\begin{array}{l}\text { 1,5 g Dermasil } \\
\text { Perfekt, Silex } \\
3000 \text { oder Triplex } \\
\text { energy PF }\end{array}$ & $\begin{array}{l}\text { 0,6 ml Ozonit } \\
\text { Performance }\end{array}$ & 70 & 10 & $1: 4$ & $A B$ & Ecolab \\
\hline Penta Aktiv-Verfahren & $4 \mathrm{~g}$ Tena ST & $1 \mathrm{~g}$ Penta-Aktiv ${ }^{\mathrm{a}}$ & 60 & 15 & $1: 4$ & A & $\begin{array}{l}\text { Burnus- } \\
\text { Hychem }\end{array}$ \\
\hline Penta Aktiv-Verfahren & $\begin{array}{l}0,5 \mathrm{~g} \text { Liquisan } \mathrm{A} \\
\text { oder Liquisan } \mathrm{A} \\
\text { aufhellerfrei und } \\
1 \mathrm{~g} \text { Liquisan } \mathrm{B}\end{array}$ & $1 \mathrm{~g}$ Penta-Aktiv ${ }^{\mathrm{a}}$ & 65 & 10 & $1: 4$ & $A B$ & $\begin{array}{l}\text { Burnus- } \\
\text { Hychem }\end{array}$ \\
\hline Penta Aktiv-Verfahren & $3 \mathrm{~g}$ Olisso Power & $1 \mathrm{~g}$ Penta-Aktiv ${ }^{\mathrm{a}}$ & 65 & 10 & $1: 4$ & $A B$ & $\begin{array}{l}\text { Burnus- } \\
\text { Hychem }\end{array}$ \\
\hline Penta Aktiv-Verfahren & $\begin{array}{l}4 \mathrm{~g} \text { Tena ST oder } \\
4 \mathrm{~g} \text { Penta-Basis }\end{array}$ & $1 \mathrm{~g}$ Penta-Aktiv ${ }^{\mathrm{a}}$ & 70 & 10 & $1: 4$ & $A B$ & $\begin{array}{l}\text { Burnus- } \\
\text { Hychem }\end{array}$ \\
\hline Penta Aktiv-Verfahren & 2,5 g Tenalan W & $1 \mathrm{~g}$ Penta-Aktiv ${ }^{\mathrm{a}}$ & 70 & 8 & $1: 4$ & $A B$ & $\begin{array}{l}\text { Burnus- } \\
\text { Hychem }\end{array}$ \\
\hline Penta Aktiv-Verfahren & $\begin{array}{l}4 \mathrm{~g} \text { Tena PF oder } \\
\text { Tena PF aufheller- } \\
\text { frei }\end{array}$ & $1 \mathrm{~g}$ Penta-Aktiv ${ }^{\mathrm{a}}$ & 70 & 10 & $1: 4$ & $A B$ & $\begin{array}{l}\text { Burnus- } \\
\text { Hychem }\end{array}$ \\
\hline Peracid-Verfahren & $4 \mathrm{~g}$ Selox Micran & $\begin{array}{l}6 \mathrm{ml} \text { Peracid } \\
\text { Forte }^{\mathrm{a}}\end{array}$ & 40 & 10 & $1: 4$ & $A B$ & $\begin{array}{l}\text { Christeyns } \\
\text { GmbH }\end{array}$ \\
\hline
\end{tabular}




\begin{tabular}{|c|c|c|c|c|c|c|c|}
\hline \multirow[t]{2}{*}{ Name } & \multicolumn{2}{|c|}{ Konzentration (g auf 1 I Flotte) } & \multirow{2}{*}{$\begin{array}{l}\text { Desinfek- } \\
\text { tionstempe- } \\
\text { ratur in }{ }^{\circ} \mathrm{C}\end{array}$} & \multirow{2}{*}{$\begin{array}{l}\text { Einwirkungs- } \\
\text { zeit in min }\end{array}$} & \multirow{2}{*}{$\begin{array}{l}\text { Flotten- } \\
\text { verhältnis }\end{array}$} & \multirow{2}{*}{$\begin{array}{l}\text { Wirkungs- } \\
\text { bereich }\end{array}$} & \multirow{2}{*}{$\begin{array}{l}\text { Hersteller } \\
\text { bzw. } \\
\text { Lieferfirma }\end{array}$} \\
\hline & Waschmittel & $\begin{array}{l}\text { Desinfektions- } \\
\text { mittel }\end{array}$ & & & & & \\
\hline Peracid-Verfahren & $\begin{array}{l}\text { 3-5 g Flüsson ex- } \\
\text { tra, Orlit PF, 3-6 g } \\
\text { Solvit spezial oder } \\
\text { Teut A spezial }\end{array}$ & $2 \mathrm{ml} \mathrm{Peracid}^{\mathrm{a}}$ & 60 & 15 & $1: 5$ & $A B$ & $\begin{array}{l}\text { Christeyns } \\
\text { GmbH }\end{array}$ \\
\hline Peracid-Verfahren & $\begin{array}{l}3-5 \mathrm{~g} \text { Orlit, } 3-5 \mathrm{~g} \\
\text { GT } 12 \text { oder Majes- } \\
\text { tic soap }\end{array}$ & $2 \mathrm{ml} \mathrm{Peracid}^{\mathrm{a}}$ & 65 & 15 & $1: 5$ & $A B$ & $\begin{array}{l}\text { Christeyns } \\
\text { GmbH }\end{array}$ \\
\hline Peracid-Verfahren & $\begin{array}{l}\text { 3-5 g Flüsson } \\
\text { extra, GT 12, Ma- } \\
\text { jestic soap, Orlit } \\
\text { PF, Solvit Spezial, } \\
\text { Teut A Spezial } \\
\text { oder 3-5 ml Flüs- } \\
\text { son Liquid }\end{array}$ & $2 \mathrm{ml} \mathrm{Peracid}^{\mathrm{a}}$ & 70 & 10 & $1: 5$ & $A B$ & $\begin{array}{l}\text { Christeyns } \\
\text { GmbH }\end{array}$ \\
\hline Peracid-Verfahren & $\begin{array}{l}1,5 \mathrm{~g} \text { Majestic } \\
\text { Maxima }\end{array}$ & $\begin{array}{l}0,6 \mathrm{ml} \text { Peracid } \\
\text { Forte }^{\mathrm{a}}\end{array}$ & 60 & 10 & $1: 5$ & $A B$ & $\begin{array}{l}\text { Christeyns } \\
\text { GmbH }\end{array}$ \\
\hline Peracid-Verfahren & $\begin{array}{l}\text { 3-5 g Orlit PF } \\
\text { oder 3-6 g Solvit } \\
\text { spezial }\end{array}$ & $\begin{array}{l}0,7 \mathrm{ml} \text { Peracid } \\
\text { Forte }^{\mathrm{a}}\end{array}$ & 60 & 15 & $1: 5$ & $A B$ & $\begin{array}{l}\text { Christeyns } \\
\text { GmbH }\end{array}$ \\
\hline Peracid-Verfahren & $\begin{array}{l}3-5 \text { g GT } 12 \text { oder } \\
\text { Majestic soap }\end{array}$ & $\begin{array}{l}0,7 \mathrm{ml} \text { Peracid } \\
\text { Forte }^{\mathrm{a}}\end{array}$ & 65 & 15 & $1: 5$ & $A B$ & $\begin{array}{l}\text { Christeyns } \\
\text { GmbH }\end{array}$ \\
\hline Peracid-Verfahren & $\begin{array}{l}\text { 1,2 g Majestic } \\
\text { classic }\end{array}$ & $\begin{array}{l}0,7 \mathrm{ml} \text { Peracid } \\
\text { Forte }^{\mathrm{a}}\end{array}$ & 70 & 10 & $1: 5$ & A & $\begin{array}{l}\text { Christeyns } \\
\text { GmbH }\end{array}$ \\
\hline Peracid-Verfahren & $\begin{array}{l}\text { 3-5 g GT 12, } \\
\text { Majestic soap, } \\
\text { Nemata MG, Orlit } \\
\text { PF, Solvit Spezial, } \\
\text { Teut A Spezial } \\
\text { oder 2,4 g Majestic } \\
\text { classic }\end{array}$ & $\begin{array}{l}0,7 \mathrm{ml} \text { Peracid } \\
\text { Forte }^{\mathrm{a}}\end{array}$ & 70 & 10 & $1: 5$ & $A B$ & $\begin{array}{l}\text { Christeyns } \\
\text { GmbH }\end{array}$ \\
\hline Per Ezet-Verfahren & $3 \mathrm{~g}$ Zeiss-Brillant & $\begin{array}{l}1 \mathrm{ml} \text { Per Ezet } \\
\text { Konz }^{\mathrm{a}}\end{array}$ & 70 & 10 & $1: 5$ & $A B$ & Ernst Zeiss \\
\hline Personril-Verfahren & $\begin{array}{l}\text { 3- } 6 \text { g Alfa, Crystal } \\
\text { classic, PC 1, Os- } \\
\text { maflux profi oder } \\
\text { Rekord }\end{array}$ & $2 \mathrm{ml}$ Personril ${ }^{\mathrm{a}}$ & 60 & 15 & $1: 5$ & $A B$ & $\begin{array}{l}\text { Christeyns } \\
\text { GmbH }\end{array}$ \\
\hline Personril-Verfahren & $2 \mathrm{~g}$ Power Activ & $1,6 \mathrm{ml}$ Personril ${ }^{\mathrm{a}}$ & 70 & 8 & $1: 4$ & $A B$ & $\begin{array}{l}\text { Christeyns } \\
\text { GmbH }\end{array}$ \\
\hline Personril-Verfahren & $\begin{array}{l}2 \text { g Majestic sava, } \\
3 \text { g Majestic, } \\
\text { Osmaflux thema } \\
\text { oder Record } \\
\text { special }\end{array}$ & 1,6 ml Personrila & 70 & 10 & $1: 4$ & $A B$ & $\begin{array}{l}\text { Christeyns } \\
\text { GmbH }\end{array}$ \\
\hline Personril-Verfahren & $\begin{array}{l}3 \text { g Majestic sava, } \\
\text { Osmaflux profi, } \\
\text { Rekord oder San }\end{array}$ & 1,6 ml Personril ${ }^{\mathrm{a}}$ & 70 & 10 & $1: 5$ & $A B$ & $\begin{array}{l}\text { Christeyns } \\
\text { GmbH }\end{array}$ \\
\hline PES 32-Verfahren & $\begin{array}{l}\text { 2-4 g Maximo } \\
\text { liquid }\end{array}$ & $2 \mathrm{ml} \mathrm{PES} 32^{\mathrm{a}}$ & 70 & 10 & $1: 5$ & A & Kleen Purgatis \\
\hline Prima Oxy-Verfahren & $\begin{array}{l}4 \mathrm{ml} \text { Prima Hy- } \\
\text { giene }\end{array}$ & $2 \mathrm{ml}$ Prima Oxy & 60 & 15 & $1: 5$ & $A B$ & Dr. Schnell \\
\hline REM PER-Verfahren & $5 \mathrm{~g}$ Waschpon & $\begin{array}{l}4 \mathrm{ml} \text { REM PER } \\
\text { Konz }^{\mathrm{a}}\end{array}$ & 40 & 20 & $1: 5$ & $A$ & van Baerle \\
\hline REM PER-Verfahren & $5 \mathrm{~g}$ Melsit super & $\begin{array}{l}2 \mathrm{ml} \text { REM PER } \\
\text { oder } 1 \mathrm{ml} \text { REM } \\
\text { PER Konz }{ }^{\mathrm{a}}\end{array}$ & 60 & 15 & $1: 5$ & $A$ & van Baerle \\
\hline
\end{tabular}




\begin{tabular}{|c|c|c|c|c|c|c|c|}
\hline \multirow[t]{2}{*}{ Name } & \multicolumn{2}{|c|}{ Konzentration (g auf 1 I Flotte) } & \multirow{2}{*}{$\begin{array}{l}\text { Desinfek- } \\
\text { tionstempe- } \\
\text { ratur in }{ }^{\circ} \mathrm{C}\end{array}$} & \multirow{2}{*}{$\begin{array}{l}\text { Einwirkungs- } \\
\text { zeit in min }\end{array}$} & \multirow{2}{*}{$\begin{array}{l}\text { Flotten- } \\
\text { verhältnis }\end{array}$} & \multirow{2}{*}{$\begin{array}{l}\text { Wirkungs- } \\
\text { bereich }\end{array}$} & \multirow{2}{*}{$\begin{array}{l}\text { Hersteller } \\
\text { bzw. } \\
\text { Lieferfirma }\end{array}$} \\
\hline & Waschmittel & $\begin{array}{l}\text { Desinfektions- } \\
\text { mittel }\end{array}$ & & & & & \\
\hline REM PER-Verfahren & 5 ml Waschpon & $\begin{array}{l}2 \mathrm{ml} \text { REM PER } \\
\text { oder } 1 \mathrm{ml} \text { REM } \\
\text { PER Konz }{ }^{\mathrm{a}}\end{array}$ & 60 & 15 & $1: 5$ & $A B$ & van Baerle \\
\hline REM PER-Verfahren & $5 \mathrm{~g}$ Melsit super & $\begin{array}{l}1 \mathrm{ml} \text { REM PER } \\
\text { Konz }^{\mathrm{a}}\end{array}$ & 60 & 15 & 1:5 & $A B$ & van Baerle \\
\hline REM PER-Verfahren & $\begin{array}{l}5 \mathrm{~g} \text { Melsit super } \\
\text { oder } 5 \mathrm{~g} \text { Wasch- } \\
\text { pon }\end{array}$ & $\begin{array}{l}1 \mathrm{ml} \text { REM PER } \\
\text { Konz }^{\mathrm{a}}\end{array}$ & 70 & 10 & $1: 5$ & $A B$ & van Baerle \\
\hline Rheosol Acid 5-Verfahren & $\begin{array}{l}\text { 2-6g Rheosol } \\
\text { Basic }\end{array}$ & $\begin{array}{l}2 \mathrm{ml} \text { Rheosol } \\
\text { Acid-5a }\end{array}$ & 60 & 15 & $1: 5$ & A & $\begin{array}{l}\text { Wachendorf- } \\
\text { Chemie }\end{array}$ \\
\hline Sanoxy Asepsis-Verfahren & $\begin{array}{l}\text { 1,5-2,5 g Majes- } \\
\text { tic classic, } 1,2 \mathrm{~g} \\
\text { Majestic matrix } \\
\text { oder } 1 \mathrm{ml} \text { Power } \\
\text { Progress }\end{array}$ & $\begin{array}{l}\text { 0,7 ml Sanoxy } \\
\text { Asepsis }\end{array}$ & $\begin{array}{l}50 \\
\text { anschl. } \\
70\end{array}$ & $\begin{array}{l}8 \\
10\end{array}$ & 1:5 & $A B$ & $\begin{array}{l}\text { Christeyns } \\
\text { GmbH }\end{array}$ \\
\hline Sanoxy Forte-Verfahren & $\begin{array}{l}1,5 \mathrm{~g} \text { Majestic } \\
\text { Maxima }\end{array}$ & $\begin{array}{l}0,6 \mathrm{ml} \text { Sanoxy } \\
\text { Forte }^{\mathrm{a}}\end{array}$ & 60 & 10 & $1: 5$ & $A B$ & $\begin{array}{l}\text { Christeyns } \\
\text { GmbH }\end{array}$ \\
\hline Sept PES-Verfahren & $\begin{array}{l}\text { 4-6 g Ozerna } 1 \\
\text { Super }\end{array}$ & $2 \mathrm{ml}$ Sept PESa & 60 & 15 & $1: 5$ & A & BÜFA \\
\hline Sept PES-Verfahren & $4 \mathrm{~g}$ Ozerna 1 Super & $2 \mathrm{ml}$ Sept PESa & 60 & 15 & $1: 5$ & $A B$ & BÜFA \\
\hline Sept PES Forte-Verfahren & $\begin{array}{l}4 \mathrm{ml} \text { Ozerna } \\
\text { Perfekt }\end{array}$ & $\begin{array}{l}2 \mathrm{ml} \text { Sept PES } \\
\text { Forte }^{\mathrm{a}}\end{array}$ & 60 & 15 & $1: 5$ & $A B$ & BÜFA \\
\hline Trebon 3-Verfahren & $2 \mathrm{~g}$ Trebon 1 & $1,5 \mathrm{ml}$ Trebon $3^{\mathrm{a}}$ & 65 & 14 & $1: 5$ & A & Kreussler \\
\hline Trisanox-Verfahren & $\begin{array}{l}1 \mathrm{~g} \text { Trisanox A und } \\
4 \mathrm{~g} \text { Trisanox B }\end{array}$ & 0,7 g Trisanox Ca & 60 & 20 & $1: 5$ & A & $\begin{array}{l}\text { Burnus- } \\
\text { Hychem }\end{array}$ \\
\hline Trisanox-Verfahren & $5 \mathrm{~g}$ Trisanox B & 0,8 $\mathrm{g}$ Trisanox $\mathrm{Ca}^{\mathrm{a}}$ & 70 & 10 & $1: 4$ & $A B$ & $\begin{array}{l}\text { Burnus- } \\
\text { Hychem }\end{array}$ \\
\hline Viva Duox-Verfahren & 1,5 g Viva Pris & 1,5 g Viva Duox ${ }^{\mathrm{a}}$ & 60 & 10 & $1: 5$ & $A B$ & Seitz \\
\hline Viva Duox-Verfahren & $1 \mathrm{~g}$ Mega Power & 1,7 g Viva Duox ${ }^{\mathrm{a}}$ & 70 & 10 & $1: 5$ & $A B$ & Seitz \\
\hline Viva Oxy-Verfahren & $\begin{array}{l}3 \mathrm{~g} \text { Mega Sol oder } \\
3 \mathrm{~g} \text { Viva Sol }\end{array}$ & 2 ml Viva Oxya & 70 & 10 & $1: 5$ & $A B$ & Seitz \\
\hline Amerah-Verfahren & $5 \mathrm{~g}$ Amerah & & 60 & 20 & $1: 5$ & $A B$ & Gustav Koch \\
\hline ARENAS-Perla-Des-Verfahren & $5 \mathrm{~g}$ ARENAS-Perla-D & & 60 & 20 & 1:5 & $A B$ & $\begin{array}{l}\text { Johannes } \\
\text { Kiehl }\end{array}$ \\
\hline $\begin{array}{l}\text { CLEAN and CLEVER Professio- } \\
\text { nal Desinfektionswaschmittel } \\
\text { PRO 38-Verfahren }\end{array}$ & $\begin{array}{l}7 \mathrm{~g} \text { CLEAN and CLE } \\
\text { Desinfektionswasch }\end{array}$ & $\begin{array}{l}\text { ER Professional } \\
\text { mittel PRO } 38\end{array}$ & 60 & 20 & $1: 5$ & $A B$ & $\begin{array}{l}\text { lgefa } \\
\text { Handels- } \\
\text { gesellschaft }\end{array}$ \\
\hline $\begin{array}{l}\text { CLEAN and CLEVER Professio- } \\
\text { nal Desinfektionswaschmittel } \\
\text { PRO 38-Verfahren }\end{array}$ & $\begin{array}{l}5 \mathrm{~g} \text { CLEAN and CLE } \\
\text { Desinfektionswasch }\end{array}$ & $\begin{array}{l}\text { ER Professional } \\
\text { mittel PRO } 38\end{array}$ & 65 & 20 & $1: 5$ & $A B$ & $\begin{array}{l}\text { Igefa Han- } \\
\text { delsgesell- } \\
\text { schaft }\end{array}$ \\
\hline Desimat-Verfahren & $5 \mathrm{~g}$ Desimat & & 60 & 20 & $1: 5$ & $A B$ & $\begin{array}{l}\text { Becker Che- } \\
\text { mie }\end{array}$ \\
\hline Desosan-Verfahren & $6 \mathrm{~g}$ Desosan & & 60 & 15 & $1: 5$ & $A B$ & $\begin{array}{l}\text { Christeyns } \\
\text { GmbH }\end{array}$ \\
\hline Desotex-Verfahren & $6 \mathrm{~g}$ Desotex & & 75 & 15 & $1: 5$ & $A B$ & $\begin{array}{l}\text { Christeyns } \\
\text { GmbH }\end{array}$ \\
\hline Desotex-Verfahren & $7 \mathrm{~g}$ Desotex & & 60 & 20 & 1:5 & A & $\begin{array}{l}\text { Christeyns } \\
\text { GmbH }\end{array}$ \\
\hline Destex-Verfahren & $5 \mathrm{~g}$ Destex & & 60 & 20 & $1: 5$ & $A B$ & $\begin{array}{l}\text { Textil Catering } \\
\text { Service }\end{array}$ \\
\hline DUROPLEX-Verfahren & $2,5 \mathrm{~g}$ DUROPLEX & & 70 & 10 & $1: 5$ & $A B$ & $\begin{array}{l}\text { BurnusHy- } \\
\text { chem }\end{array}$ \\
\hline Eltra-Verfahren & $5 \mathrm{~g}$ Eltra & & 70 & 10 & $1: 5$ & $A B$ & Ecolab \\
\hline Eltra-Verfahren & $7 \mathrm{~g}$ Eltra & & 60 & 20 & $1: 5$ & $A B$ & Ecolab \\
\hline
\end{tabular}




\begin{tabular}{|c|c|c|c|c|c|c|}
\hline \multirow[t]{2}{*}{ Name } & Konzentration (g auf 1 I Flotte) & \multirow{2}{*}{$\begin{array}{l}\text { Desinfek- } \\
\text { tionstempe- } \\
\text { ratur in }{ }^{\circ} \mathrm{C}\end{array}$} & \multirow{2}{*}{$\begin{array}{l}\text { Einwirkungs- } \\
\text { zeit in min }\end{array}$} & \multirow{2}{*}{$\begin{array}{l}\text { Flotten- } \\
\text { verhältnis }\end{array}$} & \multirow{2}{*}{$\begin{array}{l}\text { Wirkungs- } \\
\text { bereich }\end{array}$} & \multirow{2}{*}{$\begin{array}{l}\text { Hersteller } \\
\text { bzw. } \\
\text { Lieferfirma }\end{array}$} \\
\hline & $\begin{array}{l}\text { Desinfektions- } \\
\text { mittel }\end{array}$ & & & & & \\
\hline Eltra 40-Verfahren ${ }^{b}$ & $8 \mathrm{~g}$ Eltra 40 & 40 & 20 & $1: 5$ & $A B$ & Ecolab \\
\hline Gabilan Plus Verfahren & $5 \mathrm{~g}$ Gabilan Plus & 60 & 20 & $1: 5$ & $A B$ & Enzian Seifen \\
\hline Germatex-Verfahren & $7 \mathrm{~g}$ Germatex & 60 & 20 & 1:5 & $A B$ & $\begin{array}{l}\text { Burnus- } \\
\text { Hychem }\end{array}$ \\
\hline Gomesan-Verfahren & $5 \mathrm{~g}$ Gomesan & 70 & 10 & $1: 5$ & $A B$ & $\begin{array}{l}\text { Christeyns } \\
\text { GmbH }\end{array}$ \\
\hline Gomesan-Verfahren & $6 \mathrm{~g}$ Gomesan & 60 & 20 & $1: 5$ & A & $\begin{array}{l}\text { Christeyns } \\
\text { GmbH }\end{array}$ \\
\hline havonPERFECT-Verfahren & $7 \mathrm{~g}$ havonPERFECT & 60 & 20 & $1: 5$ & A & $\begin{array}{l}\text { Hagleitner } \\
\text { Hygiene }\end{array}$ \\
\hline Hexawa Hospital-Verfahren & $5 \mathrm{~g}$ Hexawa Hospital & 65 & 20 & $1: 5$ & $A B$ & Dreiturm \\
\hline Hexawa Hospital-Verfahren & $7 \mathrm{~g}$ Hexawa Hospital & 60 & 20 & $1: 5$ & A & Dreiturm \\
\hline Jumbo Hygiene-Verfahren & $5 \mathrm{~g}$ Jumbo Hygiene & 60 & 20 & $1: 5$ & $A B$ & mobiloclean \\
\hline Lavo Des 60 kompakt-Verfahren & 3,6 g Lavo Des 60 kompakt & 60 & 15 & $1: 5$ & $A B$ & Kleen Purgatis \\
\hline Lavo Des 60 plus-Verfahren & $5 \mathrm{~g}$ Lavo Des 60 plus & 60 & 20 & 1:5 & A & Kleen Purgatis \\
\hline Lloyd D 90-Verfahren & $5 \mathrm{~g}$ Lloyd D 90 & 70 & 10 & 1:5 & $A B$ & $\begin{array}{l}\text { August } \\
\text { Wencke }\end{array}$ \\
\hline Lloyd D 90-Verfahren & 6 g Lloyd D 90 & 60 & 20 & $1: 5$ & A & $\begin{array}{l}\text { August } \\
\text { Wencke }\end{array}$ \\
\hline Lunosan-Verfahren & $7 \mathrm{~g}$ Lunosan & 60 & 20 & $1: 5$ & A & $\begin{array}{l}\text { Christeyns } \\
\text { GmbH }\end{array}$ \\
\hline Mega Pur-Verfahren & $5 \mathrm{~g}$ Mega Pur & 60 & 20 & $1: 5$ & $A B$ & Seitz \\
\hline Monosan-Verfahren & $7 \mathrm{~g}$ Monosan & 60 & 20 & $1: 5$ & $A B$ & $\begin{array}{l}\text { Burnus- } \\
\text { Hychem }\end{array}$ \\
\hline Monosan PF-Verfahren & $6 \mathrm{~g}$ Monosan PF & 60 & 20 & $1: 5$ & $A B$ & $\begin{array}{l}\text { Burnus- } \\
\text { Hychem }\end{array}$ \\
\hline mopEltra-Verfahren & $6 \mathrm{~g} \mathrm{mopEltra}$ & 60 & 15 & $1: 5$ & $A B$ & Ecolab \\
\hline Oxyplex Perfekt-Verfahren & $5 \mathrm{~g}$ Oxyplex Perfekt & 65 & 20 & $1: 5$ & $A B$ & $\begin{array}{l}\text { Burnus- } \\
\text { Hychem }\end{array}$ \\
\hline Oxyplex Perfekt-Verfahren & 7 g Oxyplex Perfekt & 60 & 20 & $1: 5$ & $A B$ & $\begin{array}{l}\text { Burnus- } \\
\text { Hychem }\end{array}$ \\
\hline Ozerna Sept-Verfahren & $5 \mathrm{~g}$ Ozerna Sept & 65 & 20 & $1: 5$ & $A B$ & BÜFA \\
\hline Ozerna Sept-Verfahren & $7 \mathrm{~g}$ Ozerna Sept & 60 & 20 & $1: 5$ & A & BÜFA \\
\hline $\begin{array}{l}\text { Persil Desinfektions- } \\
\text { Vollwaschmittel-Verfahren }\end{array}$ & $\begin{array}{l}3,6 \mathrm{~g} \text { Persil Desinfektions-Vollwasch- } \\
\text { mittel }\end{array}$ & 60 & 15 & $1: 5$ & $A B$ & Henkel \\
\hline Perlweiss D-Verfahren & 7 g Perlweiss D & 60 & 20 & $1: 5$ & $A B$ & Seeger \\
\hline RAPA plus-Verfahren & $6 \mathrm{~g}$ RAPA plus & 60 & 20 & $1: 5$ & $A B$ & $\begin{array}{l}\text { Dr. Schnell } \\
\text { Chemie }\end{array}$ \\
\hline Rheosol Deso-Verfahren & $5 \mathrm{~g}$ Rheosol Deso & 60 & 20 & $1: 5$ & A & $\begin{array}{l}\text { Wachendorf- } \\
\text { Chemie }\end{array}$ \\
\hline Roland HY 90-Verfahren & $5 \mathrm{~g}$ Roland HY 90 & 70 & 10 & $1: 5$ & $A B$ & $\begin{array}{l}\text { August } \\
\text { Wencke }\end{array}$ \\
\hline Roland HY 90-Verfahren & $6 \mathrm{~g}$ Roland HY 90 & 60 & 20 & 1:5 & $A$ & $\begin{array}{l}\text { August } \\
\text { Wencke }\end{array}$ \\
\hline Sanomat-Verfahren & $5 \mathrm{~g}$ Sanomat & 60 & 20 & $1: 5$ & $A B$ & Rösch \\
\hline Saponmatic Hygiene-Verfahren & $5 \mathrm{~g}$ saponmatic Hygiene & 60 & 20 & $1: 5$ & $A B$ & Rösch \\
\hline Supersan forte-Verfahren & $7 \mathrm{~g}$ Supersan forte & 60 & 20 & $1: 5$ & $A B$ & HIMED \\
\hline
\end{tabular}




\begin{tabular}{|c|c|c|c|c|c|c|c|}
\hline \multirow[t]{2}{*}{ Name } & \multicolumn{2}{|c|}{ Konzentration (g auf 1 I Flotte) } & \multirow{2}{*}{$\begin{array}{l}\text { Desinfek- } \\
\text { tionstempe- } \\
\text { ratur in }{ }^{\circ} \mathrm{C}\end{array}$} & \multirow{2}{*}{$\begin{array}{l}\text { Einwirkungs- } \\
\text { zeit in min }\end{array}$} & \multirow{2}{*}{$\begin{array}{l}\text { Flotten- } \\
\text { verhältnis }\end{array}$} & \multirow{2}{*}{$\begin{array}{l}\text { Wirkungs- } \\
\text { bereich }\end{array}$} & \multirow{2}{*}{$\begin{array}{l}\text { Hersteller } \\
\text { bzw. } \\
\text { Lieferfirma }\end{array}$} \\
\hline & Waschmittel & $\begin{array}{l}\text { Desinfektions- } \\
\text { mittel }\end{array}$ & & & & & \\
\hline Thermoton Washtex-Verfahren & \multicolumn{2}{|c|}{$5 \mathrm{~g}$ Thermoton Washtex } & 60 & 20 & $1: 5$ & $A B$ & $\begin{array}{l}\text { Dr. Schuma- } \\
\text { cher }\end{array}$ \\
\hline Trebon Plus-Verfahren & \multicolumn{2}{|l|}{$4 \mathrm{~g}$ Trebon Plus } & 60 & 20 & $1: 5$ & $A B$ & Kreussler \\
\hline Zack Astramatic Hygiene-Verfahren & \multicolumn{2}{|c|}{$6 \mathrm{~g}$ Zack Astramatic Hygiene } & 60 & 20 & $1: 5$ & A & $\begin{array}{l}\text { August } \\
\text { Wencke }\end{array}$ \\
\hline Zack Astramatic Hygiene-Verfahren & \multicolumn{2}{|c|}{$5 \mathrm{~g}$ Zack Astramatic Hygiene } & 70 & 10 & $1: 5$ & $A B$ & $\begin{array}{l}\text { August } \\
\text { Wencke }\end{array}$ \\
\hline
\end{tabular}

\begin{tabular}{|c|c|c|c|c|c|c|c|}
\hline \multirow[t]{2}{*}{ Name } & \multicolumn{2}{|c|}{ Konzentration (g auf 1 I Flotte) } & \multirow{2}{*}{$\begin{array}{l}\text { Desinfektions- } \\
\text { temperatur } \\
\text { in }{ }^{\circ} \mathrm{C}\end{array}$} & \multirow{2}{*}{$\begin{array}{l}\text { Einwirkungszeit } \\
\text { in } \min \end{array}$} & \multirow{2}{*}{$\begin{array}{l}\text { Flottenverhält- } \\
\text { nis }\end{array}$} & \multirow{2}{*}{$\begin{array}{l}\text { Wirkungsbe- } \\
\text { reich }\end{array}$} & \multirow{2}{*}{$\begin{array}{l}\text { Hersteller bzw. } \\
\text { Lieferfirma }\end{array}$} \\
\hline & Waschmittel & $\begin{array}{l}\text { Desinfek- } \\
\text { tionsmittel }\end{array}$ & & & & & \\
\hline Saniton-Verfahren & $\begin{array}{l}5 \mathrm{~g} \text { Melsit super } \\
\text { oder Waschpon } \\
\text { universal }\end{array}$ & $\begin{array}{l}0,6 \mathrm{~g} \\
\text { Saniton }^{\mathrm{a}}\end{array}$ & 60 & 10 & $1: 5$ & $A B$ & van Baerle \\
\hline Trixon-Verfahren & $\begin{array}{l}\text { 3-6 g Aliplex, } \\
\text { Osetta, oder } \\
\text { Uniplex }\end{array}$ & $\begin{array}{l}0,6 \mathrm{~g} \\
\text { Trixon }^{\mathrm{a}}\end{array}$ & 60 & 10 & $1: 5$ & $A B$ & BurnusHychem \\
\hline
\end{tabular}

\subsection{Instrumentendes- infektion in Reinigungs- und Desinfektionsgeräten}

Die im Folgenden aufgeführten Geräte wurden gemäß der „Prüfrichtlinie des Bundesgesundheitsamtes zur Prüfung von thermischen Desinfektionsverfahren in Reinigungsautomaten " [Bundesgesundheitsbl. (1980) 23:36-367] geprüft. Hierbei stand die desinfizierende Wirkung im Vordergrund, die Reinigungsleistung wurde nicht geprüft. Auf die „Mitteilung des Robert Koch-Instituts zur Aufnahme von Reinigungs- und Desinfektionsgeräten in die Liste der geprüften und anerkannten Desinfektionsmittel und -verfahren gemäß $\$ 18$ IfSG“ [Bundesgesundheitsbl. (2007) 50:128129] wird ausdrücklich hingewiesen. Die Wirksamkeit wurde jeweils nur für das in die Liste eingetragene Programm nachgewiesen. Bei der Aufbereitung von $\mathrm{Me}$ dizinprodukten ist die Empfehlung der Kommission für Krankenhaushygiene und Infektionsprävention beim RKI und des Bundesinstitutes für Arzneimittel und Medizinprodukte „Anforderungen an die Hygiene bei der Aufbereitung von Medizinprodukten" [Bundesgesundheitsbl. 55 (2012):1244-1310] zu beachten.
Die Bedienungs- und Beladungsvorschriften der Hersteller sind zu beachten. Es sollten nur die vom Hersteller des Reinigungsautomaten für die jeweiligen Anwendungszwecke empfohlenen Reinigungsmittel verwendet werden. Während der Desinfektionsphase darf keine Flotte aus der Maschine austreten. Der Desinfektionsvorgang muss vor dem erstmaligen Ablassen der Flotte abgeschlossen sein. Die Abluft ist so abzuführen bzw. nachzubehandeln, dass von ihr keine Gefahr ausgehen kann.

Bei der Angabe der Desinfektionstemperatur handelt es sich um den oberen Schaltpunkt des Thermostaten des jeweiligen Gerätes. Es soll damit gewährleistet werden, dass während der Einwirkungszeit eine Temperatur von $90^{\circ} \mathrm{C}$ nicht unterschritten wird.

Für Instrumente mit langen bzw. engen Hohlräumen sind die Verfahren nur dann geeignet, wenn diese Hohlräume von der heißen Flotte durchströmt werden. Bei Reinigungsautomaten mit speziellen Instrumentenanschlüssen sind die nicht genutzten Düsen dicht zu verschließen, um einen ausreichenden Spüldruck zu gewährleisten.

\subsubsection{System Belimed}

Thermisches Desinfektions- und Reinigungsverfahren für Instrumente, Laborglas und Zubehör von Anästhesiegeräten

\section{Betriebsdaten}

Desinfektionstemperatur: $93^{\circ} \mathrm{C}$

Einwirkungszeit: $10 \mathrm{~min}$

Wirkungsbereich: AB

Geprüfte und anerkannte Apparatetypen: WD 100, WD 130, WD 170, WD 220, WD 280

\subsubsection{System BHT Hygiene Technik}

Thermisches Desinfektions- und Reinigungsverfahren für Instrumente, Laborglas und Zubehör von Anästhesiegeräten

\section{Betriebsdaten}

Desinfektionstemperatur: $93^{\circ} \mathrm{C}$

Einwirkungszeit: $10 \mathrm{~min}$

Wirkungsbereich: AB

Geprüfte und anerkannte Apparatetypen: INNOVA M 3, INNOVA M 4, INNOVA M 5 


\subsubsection{System Lancer}

Thermisches Desinfektions- und Reinigungsverfahren für Instrumente, Laborglas und Zubehör von Anästhesiegeräten

\section{Betriebsdaten}

Desinfektionstemperatur: $93^{\circ} \mathrm{C}$

a) Einwirkungszeit: $1 \mathrm{~min}$

Wirkungsbereich: A

Geprüfter und anerkannter Apparatetyp: 820 UP

b) Einwirkungszeit: 10 min

Wirkungsbereich: $\mathrm{AB}$

Geprüfte und anerkannte Apparatetypen: HOSPITALIA 520, 820 UP

\subsubsection{System Riebesam}

Thermisches Desinfektions- und Reinigungsverfahren für Instrumente, Laborglas und Zubehör von Anästhesiegeräten

\section{Betriebsdaten}

Desinfektionstemperatur: $93^{\circ} \mathrm{C}$

Einwirkungszeit: $10 \mathrm{~min}$

Wirkungsbereich: $\mathrm{AB}$

Geprüfte und anerkannte Apparatetypen: $25 \mathrm{TD}, 26 \mathrm{TD}$

\subsection{Raumdesinfektion}

Eine Raumdesinfektion beinhaltet die umfassende und gleichzeitige Desinfektion aller in einem umschlossenen Raum befindlichen Oberflächen durch Verdampfen oder Vernebeln eines Desinfektionsmittels. Zusätzlich zur Raumdesinfektion ist jeweils eine Flächendesinfektion durch Wischen erforderlich. Dieses Verfahren ist nur anzuwenden, wenn besondere Infektionsgefahren bestehen und/ oder anzunehmen ist, dass die Wischdesinfektion allein unzureichend sein könnte.

\subsubsection{Verdampfung oder Vernebelung von verdünnten Formaldehyd-Lösungen mit geeigneten Apparaten}

Dosierung: $5 \mathrm{~g}$ Formaldehyd pro $\mathrm{m}^{3}$

Rauminhalt

Relative Luftfeuchtigkeit: mindestens 70\%

Einwirkungszeit: $6 \mathrm{~h}$

Wirkungsbereich: $\mathrm{AB}$
Um die vorgeschriebene Luftfeuchtigkeit zu gewährleisten, muss eine wässrige Formaldehyd-Lösung verdampft werden (pro $\mathrm{m}^{3}$ Rauminhalt z. B. $50 \mathrm{ml}$ einer 12\%igen Formaldehyd-Lösung).

Es empfiehlt sich, nach der Desinfektion den Formaldehyd durch Verdampfen von mind. $10 \mathrm{ml}$ 25\%iger Ammoniaklösung pro $\mathrm{m}^{3}$ Rauminhalt zu neutralisieren.

Bei der Durchführung der Raumdesinfektion ist die Technische Regel für Gefahrstoffe „Raumdesinfektion mit Formaldehyd“ (TRGS 522) zu beachten.

\subsubsection{Wasserstoffperoxid-Verfahren}

Begasungsverfahren mit Wasserstoffperoxid können nur bei optisch sauberen Oberflächen angewandt werden. Im Unterschied zum Formaldehydverfahren ist eine allgemeingültige Eintragung von Verfahren mit Wasserstoffperoxid für die Raumdesinfektion nicht möglich, da die Wirksamkeit dieser Verfahren von den konkreten Bedingungen vor Ort, der Art des Verfahrens sowie dem speziellen Apparat (Generator) abhängig ist. Daher ist vor der Anwendung von Raumbegasungsverfahren mit Wasserstoffperoxid für die spezifische Anwendung, d. h. für den definierten Raum, das spezielle Verfahren und den zugehörigen Apparat die Wirksamkeit zu validieren. Die Validierung setzt eine detaillierte Arbeitsanweisung voraus und umfasst mindestens die nachfolgend beschriebenen Untersuchungen [modifiziert nach Hyg Med 35 (2010):204-208]:

- Die physikalischen Parameter (Temperatur, \% rel. Feuchte, Wasserstoffperoxidkonzentration) im Raum und die Betriebsdaten des Generators für die Konditionierung (ggf. Entfeuchtung), Desinfektion und Belüftung für einen kompletten Begasungszyklus müssen mit geeigneten Prüfungen ermittelt und festgelegt werden.

- Die Prüfungen müssen auch die Ermittlung der Grenzen des Verfahrens einschließen:

- die vom Begasungsmittel im Raum am schwierigsten zu erreichenden Stellen sind mit geeigneten Indikatoren zu ermitteln;

- die erforderliche gleichmäßige Gasverteilung ist nachzuweisen;
- der Aufstellort des Generators und die Verwendung eventueller Hilfsmittel zu besseren Verteilung des Wasserstoffperoxids (Ventilatoren) sind festzulegen;

- die Inaktivierung von Bioindikatoren mit Sporen des Geobacillus stearothermophilus (s. DIN EN ISO 11138) auf allen relevanten und schwierig zu inaktivierenden Trägermaterialien (Filterpapier, Metall), auch ggf. in Schutzkolloiden (z. B. Blut) und an den vom Begasungsmittel am schwierigsten zu erreichenden Stellen im Raum ist nachzuweisen (Wirkungsbereich: $\mathrm{ABCD}$ ).

- Es muss ausgeschlossen werden, dass in dem zu begasenden Raum Materialien vorhanden sind, die Wasserstoffperoxid durch Ab- bzw. Adsorption oder Katalyse zerstören und somit den Desinfektionserfolg negativ beeinflussen.

- Die Reproduzierbarkeit des Verfahrens muss z. B. durch Standardisierung und fortlaufende Kontrolle der in der oben angegebenen Vorschrift festgelegten prozessrelevanten Parameter nachgewiesen werden.

- Es muss nachgewiesen werden, dass die Restgaskonzentration nach Belüftung unter dem vorgeschriebenen Wert $(<1 \mathrm{ppm})$ liegt.

- Der Validierung muss eine Gefährdungsanalyse in Bezug auf Personenschutz, technische Sicherheitsvorschriften, Dichtheitsprüfung des Raumes und der Fluchtwegsituation vorausgehen. Die Vorgehensweise ist auch mit den lokalen Überwachungsbzw. Genehmigungsbehörden abzustimmen.

Das Verfahren darf nur mit den bei der Validierung festgelegten Parametern durchgeführt werden, für die die Wirksamkeit nachgewiesen wurde.

Die spezifische Validierung der Wirksamkeit und die anschließende praktische Durchführung darf nur von diesbezüglich ausreichend qualifizierten Personen durchgeführt werden.

Über die Möglichkeit der Validierung nur eines von mehreren bezüglich Geometrie, Materialien und sonstiger physi- 
kalischer Parameter identischer Räume ist im Einzelfall zu entscheiden.

\subsection{Desinfektion von Abfällen}

Zur Desinfektion von Abfällen sind thermische Verfahren zu verwenden.

\subsubsection{Verbrennen}

(Wirkungsbereich: ABCD)

\subsubsection{Kochen mit Wasser}

\subsubsection{System Drauschke}

Desinfektion von Organabfällen und infektiösen Abfällen aller Art (insbesondere sog. Nassabfälle) in Wasser von mindestens $134^{\circ} \mathrm{C}$. Die Behandlung erfolgt in einem geschlossenen System ohne Luftentfernung, unter indirekter Beheizung und unter ständigem Rühren durch ein indirekt beheiztes Rührwerk.

Die bei der Eintragung vom Robert Koch-Institut erteilten Auflagen sind zu beachten.

Einwirkungszeit: $20 \mathrm{~min}$

Trocknung der Abfälle unter weiterer indirekter Beheizung und Umwälzung

Wirkungsbereich: ABCD

Geprüfter und anerkannter Apparatetyp: KSD 3000

\subsubsection{Dampfdesinfektionsverfahren}

\subsubsection{Dampfströmungsver- fahren in Apparaten gemäß DIN 58949 bzW. DIN EN 285}

Diese Verfahren sind nur geeignet für flüssige Abfälle bzw. Abfälle, die ausreichend Wasser enthalten, z. B. mikrobiologische Kulturen. Der Abfall muss in Behältern mit weiter Öffnung ohne weitere Verpackung so in die Kammer eingebracht werden, dass der Dampfzutritt zu allen Teilen des Abfalls gewährleistet ist.

Die Einwirkungszeit rechnet von dem Zeitpunkt an, zu dem alle Teile des Abfalls gesättigtem Wasserdampf ausgesetzt sind und die Desinfektionstemperatur angenommen haben.

Geeignet sind sog. Dampftöpfe bzw. Dampfdesinfektionsapparate gemäß DIN 58949 Teil 2 oder Dampfsterilisatoren ge- mäß DIN EN 285. Es sind die unter Ziffer 1.3 gegebenen Hinweise zu beachten.

\subsubsection{Dampfströmungsver- fahren in speziellen Apparaten}

\subsection{System Engstler \& Ott}

Das Verfahren ist gekennzeichnet durch:

1. Zerkleinerung des Gutes im geschlossenen System

2. Desinfektion des zerkleinerten Gutes in einem Kettenförderer mittels Sattdampf

Die bei der Eintragung vom Robert KochInstitut erteilten Auflagen, insbesondere bezüglich der Maßnahmen bei Betriebsende und Betriebsstörungen, sind zu beachten.

\section{Betriebsdaten}

Desinfektionstemperatur: $105^{\circ} \mathrm{C}$

Einwirkungszeit: $15 \mathrm{~min}$

Wirkungsbereich: ABC

Geprüfter und anerkannter Apparatetyp: ZDA-M3, Typ II

\subsubsection{Fraktionierte Vakuum-Verfahren}

Es sind die unter Ziffer 1.3.2 gegebenen Hinweise zu beachten.

\subsection{Verfahren nach}

\section{Ziffern 1.3.2.1 bis 1.3.2.5}

Die unter Ziffer 1.3.2.1 bis 1.3.2.5 aufgeführten Verfahren sind auch zur Desinfektion von Abfällen geeignet, wenn folgende Voraussetzungen erfüllt sind:

a) Die Behältnisse, in denen sich die Abfälle befinden, dürfen während der Behandlung in der Desinfektionskammer nicht luftdicht verschlossen sein. Es dürfen nur Behältnisse mit ausreichend großen Öffnungen oder Säcke verwendet werden.

b) Werden als Behältnisse Säcke verwendet, so müssen sie so beschaffen sein, dass sie - falls sie verschlossen sind - während der ersten Vakuumphase zerreißen.

c) In dem zu desinfizierenden Gut dürfen sich keine hermetisch verschlossenen Gefäße befinden, es sei denn, sie enthalten Wasser oder wässrige
Lösungen. Die Flüssigkeitsmenge pro Gefäß darf jedoch nur so groß sein, dass die Ausgleichszeit ausreicht, um die gesamte Menge auf die Desinfektionstemperatur zu erwärmen.

d) Die Ausgleichszeit und die Abkühlzeit sind auf die Abfallart abzustimmen. Dabei sind insbesondere die kompakten Bestandteile und die Flüssigkeitsmenge zu berücksichtigen. Bei der Desinfektionstemperatur von $105^{\circ} \mathrm{C}$ ist eine Einwirkungszeit von mindestens 30 min vorzusehen.

e) Die Abluft und das Kondensat sind gemäß DIN 58949 Teil 2 nachzubehandeln.

f) Die Wirksamkeit muss durch eine außerordentliche Prüfung mit der Prüfbeladung „Hohlkörper “ bestätigt werden [s. Richtlinie „Prüfung von Abfalldesinfektionsverfahren auf Wirksamkeit“, Bundesgesundheitsbl 36 (1993):158-160 bzw. DIN 58949 Teil 3].

\subsection{System Belimed Sauter}

Die bei der Eintragung vom Robert KochInstitut erteilten Auflagen, insbesondere bezüglich der Art des Abfalls und seiner Verpackung, sind zu beachten.

\section{Betriebsdaten}

Programm: Abfälle $105^{\circ} \mathrm{C}, 30 \mathrm{~min}$

Luftentfernung

Anzahl der Evakuierungsphasen: 5

In den Evakuierungsphasen zu erreichender Druck:

1. Phase: $\leq 80$ mbar

2. bis 5 . Phase: $\leq 120$ mbar

Bei den Zwischendampfstößen zu erreichender Druck: $\geq 1050$ mbar

Desinfektion

Dampftemperatur: $105^{\circ} \mathrm{C}$

Einwirkungszeit: $30 \mathrm{~min}$

Wirkungsbereich: ABC

Geprüfter und anerkannter Apparatetyp: DDA 4010

\subsection{System CMB}

Die bei der Eintragung vom Robert KochInstitut erteilten Auflagen, insbesondere 
bezüglich der Art des Abfalls und seiner

Verpackung, sind zu beachten.

\section{Betriebsdaten}

a) Programm für nicht näher spezifizierte Krankenhausabfälle

\section{Luftentfernung}

Anzahl der Evakuierungsphasen: 4

In den Evakuierungsphasen zu erreichen-

der Druck:

1. Phase: $\leq 150$ mbar

2. Phase: $\leq 200$ mbar

3. Phase: $\leq 300$ mbar

4. Phase: $\leq 300$ mbar

Bei den Zwischendampfstößen zu erreichender Druck:

1. Zwischendampfstoß: $\geq 1500$ mbar

2. Zwischendampfstoß: $\geq 1800 \mathrm{mbar}$

3. Zwischendampfstoß: $\geq 2100$ mbar

\section{Desinfektion}

Druck in der Desinfektionskammer: 2100 mbar

Einwirkungszeit: $6 \mathrm{~min}$

Wirkungsbereich: ABC

b) Programm für flüssige Abfälle

Luftentfernung

Anzahl der Evakuierungsphasen: 1

In der Evakuierungsphase zu erreichender Druck: $\leq 150$ mbar

\section{Desinfektion}

Druck in der Desinfektionskammer:

2250 mbar

Einwirkungszeit: $12 \mathrm{~min}$

Wirkungsbereich: ABC

c) Programm für flüssige Abfälle in Beuteln

\section{Luftentfernung}

Anzahl der Evakuierungsphasen: 2

In den Evakuierungsphasen zu erreichender Druck:

1. Phase: $\leq 150$ mbar

2. Phase: $\leq 300$ mbar

Bei dem Zwischendampfstoß zu erreichender Druck: $\geq 1200$ mbar
Desinfektion

Druck in der Desinfektionskammer:

2100 mbar

Einwirkungszeit: $12 \mathrm{~min}$

Wirkungsbereich: ABC

Geprüfter und anerkannter Apparatetyp:

Sintion 1.1

\subsection{System Drauschke}

Die bei der Eintragung vom Robert KochInstitut erteilten Auflagen, insbesondere bezüglich der Art des Abfalls und seiner Verpackung, sind zu beachten.

\section{Betriebsdaten}

\section{Luftentfernung}

Vor der ersten Evakuierungsphase wird bei gleichzeitiger Mantelheizung Dampf in die Kammer bis zu einem Druck von $\geq 950$ mbar eingegeben. Dieser Druck wird 15 min gehalten.

Anzahl der Evakuierungsphasen: 4

In den Evakuierungsphasen zu erreichender Druck: $\leq 100$ mbar

Bei den Zwischendampfstößen zu erreichender Druck: $\geq 1000$ mbar

Desinfektion

Dampftemperatur: $110^{\circ} \mathrm{C}$

Einwirkungszeit: $15 \mathrm{~min}$

Wirkungsbereich: ABC

Geprüfte und anerkannte Apparatetypen: MD 10, MD 13

\subsection{System Holzner}

Die bei der Eintragung vom Robert KochInstitut erteilten Auflagen, insbesondere bezüglich der Art des Abfalls und seiner Verpackung, sind zu beachten.

\section{Betriebsdaten}

a) Programm: $105^{\circ} \mathrm{C}$

Luftentfernung

Anzahl der Evakuierungsphasen: 6

In den Evakuierungsphasen zu erreichen-

der Druck:

1. Phase: $\leq 70$ mbar

2. bis 6. Phase: $\leq 120$ mbar

Bei den Zwischendampfstößen zu erreichender Druck: $\geq 1400$ mbar
Desinfektion

Dampftemperatur: $105^{\circ} \mathrm{C}$

Einwirkungszeit: $30 \mathrm{~min}$

Wirkungsbereich: ABC

b) Programm: Flüssigkeiten $121^{\circ} \mathrm{C}$

Luftentfernung

Anzahl der Evakuierungsphasen: 1

In der Evakuierungsphase zu erreichender Druck: $\leq 100$ mbar

Desinfektion

Dampftemperatur: $121^{\circ} \mathrm{C}$

Einwirkungszeit (nach Erreichen von $121^{\circ} \mathrm{C}$ an einem Temperaturfühler in dem Gut bzw. in einem Referenzgefäß. Das Referenzgefäß muss sich innerhalb des Behälters, der zur Aufnahme des Gutes dient, befinden): $20 \mathrm{~min}$

Wirkungsbereich: ABC

c) Programm: $134^{\circ} \mathrm{C}$

Luftentfernung

Anzahl der Evakuierungsphasen: 6

In den Evakuierungsphasen zu erreichender Druck:

1. Phase: $\leq 70$ mbar

2. bis 6 . Phase: $\leq 120$ mbar

Bei den Zwischendampfstößen zu erreichender Druck: $\geq 1400$ mbar

Desinfektion

Dampftemperatur: $134^{\circ} \mathrm{C}$

Einwirkungszeit: $10 \mathrm{~min}$

Wirkungsbereich: ABC

Geprüfte und anerkannte Apparatetypen:

DSLV 50.70, DSL 3.3.6, DSL 4.4.6, DSL 6.6.6, DSL 6.6.9-1, DSL 9.6.6-1-DE, DSL 9.6.9, DSL 9.6.12, DSL 12.6.12, DSL 12.9.12, DSL 14.7.14, DSL 14.8.16, DSL 18.13.15

\subsection{System HP Medizintechnik}

Die bei der Eintragung vom Robert KochInstitut erteilten Auflagen, insbesondere bezüglich der Art des Abfalls und seiner Verpackung, sind zu beachten.

\section{Betriebsdaten}

a) Programm: C-Müll fest $134^{\circ} \mathrm{C}$

Luftentfernung

Anzahl der Evakuierungsphasen: 4

In den Evakuierungsphasen zu erreichender Druck:

1. Phase: $\leq 65$ mbar

2. Phase: $\leq 70$ mbar 
3. und 4. Phase: $\leq 150$ mbar

Bei den Zwischendampfstößen zu erreichender Druck: $\geq 1250$ mbar

\section{Desinfektion}

Dampftemperatur: $134^{\circ} \mathrm{C}$

Einwirkungszeit: $20 \mathrm{~min}$

Wirkungsbereich: ABC

b) Programm: C-Müll flüssig $121^{\circ} \mathrm{C}$

Luftentfernung

Anzahl der Evakuierungsphasen: 1

In der Evakuierungsphase zu erreichender Druck: $\leq 70$ mbar

\section{Desinfektion}

Dampftemperatur: $121^{\circ} \mathrm{C}$

Einwirkungszeit: $20 \mathrm{~min}$

Wirkungsbereich: ABC

Geprüfte und anerkannte Apparatetypen: Varioklav 75 S, Varioklav 135 S

\subsection{System Lautenschläger}

Die bei der Eintragung vom Robert KochInstitut erteilten Auflagen, insbesondere bezüglich der Art des Abfalls und seiner Verpackung, sind zu beachten.

\section{Betriebsdaten}

a) Programm: Abfälle $134^{\circ} \mathrm{C}$

Luftentfernung

Anzahl der Evakuierungsphasen: 7

In den Evakuierungsphasen zu erreichender Druck:

1. bis 3. Phase: $\leq 130$ mbar

4. bis 7. Phase: $\leq 1400$ mbar

Bei den Zwischendampfstößen zu erreichender Druck:

1. und 2. Dampfstoß: $\geq 1300$ mbar

3. bis 6. Dampfstoß: $\geq 2800 \mathrm{mbar}$

\section{Desinfektion}

Dampftemperatur: $134^{\circ} \mathrm{C}$

Einwirkungszeit: $15 \mathrm{~min}$

Wirkungsbereich: ABC

b) Programm: Flüssige Abfälle $121^{\circ} \mathrm{C}$

Luftentfernung

Anzahl der Evakuierungsphasen: 5

In den Evakuierungsphasen zu erreichender Druck:

1. Phase: $\leq 50$ mbar

2. Phase: $\leq 640$ mbar
3. Phase: $\leq 860$ mbar

4. Phase: $\leq 1070$ mbar

5. Phase: $\leq 1300$ mbar

Bei den Zwischendampfstößen zu erreichender Druck:

1. und 2. Dampfstoß: $\geq 1200$ mbar

3. Dampfstoß: $\geq 1400 \mathrm{mbar}$

4. Dampfstoß: $\geq 1800$ mbar

5. Dampfstoß: $\geq 2500$ mbar

Anschließend Absenken auf den Druck, der einer Dampftemperatur von $121^{\circ} \mathrm{C}$ entspricht.

Desinfektion

Dampftemperatur: $121^{\circ} \mathrm{C}$

Einwirkungszeit: $45 \mathrm{~min}$

Wirkungsbereich: ABC

Geprüfte und anerkannte Apparatetypen: Labocert 1600, Labocert 3000, Labocert 5000

\subsection{System Miele}

Die bei der Eintragung vom Robert KochInstitut erteilten Auflagen, insbesondere bezüglich der Art des Abfalls und seiner Verpackung, sind zu beachten.

Ist der Abfall in tiefgezogenen Behältern aus Polystyrol KR 2797 verpackt, muss dem Verfahren eine zusätzliche Evakuierungsstufe auf 200 mbar und ein Dampfeinlass bis zum Erreichen von einer Temperatur von mindestens $100^{\circ} \mathrm{C}$ vorangehen.

\section{Betriebsdaten}

Luftentfernung

Anzahl der Evakuierungsphasen: 3

In den Evakuierungsphasen zu erreichender Druck:

1. Phase: $\leq 50$ mbar

2. und 3. Phase: $\leq 300$ mbar

Während der 1. Evakuierungsphase wird nach Erreichen von 50 mbar Dampf in die Kammer bis zu einem Druck von 400 mbar eingegeben und evakuiert bis auf 50 mbar

Bei den Zwischendampfstößen zu erreichender Druck: $\geq 1000$ mbar

\section{Desinfektion}

a) Dampftemperatur: $105^{\circ} \mathrm{C}$

Einwirkungszeit: $25 \mathrm{~min}$

Wirkungsbereich: ABC b) Dampftemperatur: $115^{\circ} \mathrm{C}$

Einwirkungszeit: $20 \mathrm{~min}$

Wirkungsbereich: ABC

Geprüfte und anerkannte Apparatetypen: DGD 8801, DGD 8802, DGD 8803, DGD 8804, DGD 8805

\subsection{System MMM}

Die bei der Eintragung vom Robert KochInstitut erteilten Auflagen, insbesondere bezüglich der Art des Abfalls und seiner Verpackung - Verpackungsart 1 und 2 -, sind zu beachten. Bei Verpackungsart 2 wird vor der eigentlichen Luftentfernung die Kammer auf $\leq 800$ mbar evakuiert. Danach erfolgt eine Dampfvorbehandlung der Behältnisse bei $103^{\circ} \mathrm{C}$ mit einer Haltezeit von mindestens einer Minute.

\section{Betriebsdaten}

Luftentfernung

Anzahl der Evakuierungsphasen: 5

In den Evakuierungsphasen zu erreichender Druck:

1. Phase: $\leq 80$ mbar

Folgende Phasen: $\leq 200$ mbar

Bei den Zwischendampfstößen zu erreichender Druck:

Verpackungsart 1: $\geq 1000$ mbar

Verpackungsart 2: $\geq 1250$ mbar

\section{Desinfektion}

a) Dampftemperatur: $105^{\circ} \mathrm{C}$

Einwirkungszeit: $30 \mathrm{~min}$

Wirkungsbereich: ABC

Geprüfte und anerkannte Apparatetypen: DES 1500/1501, DES 2000/2001, DES 3000/3001, DES 4000/4001, DES 6000/6001 sowie die baugleichen Typen Vacudes

b) Dampftemperatur: $134^{\circ} \mathrm{C}$

Einwirkungszeit: $10 \mathrm{~min}$

Wirkungsbereich: ABC

Geprüfte und anerkannte Apparatetypen: MLD 666, MLD 669, MLD 969, MLD 9612, MLD 12912, MLD 12924, MLD 141114, MLD 141128, MLD 181015 sowie die baugleichen Typen Monachia, Vakulab und Ventilab 


\subsection{System Ringeisen}

Die bei der Eintragung vom Robert KochInstitut erteilten Auflagen, insbesondere bezüglich der Art des Abfalls und seiner Verpackung, sind zu beachten.

\section{Betriebsdaten}

\section{Luftentfernung}

Anzahl der Evakuierungsphasen: 4

In den Evakuierungsphasen zu erreichender Druck: $\leq 50$ mbar

Bei den Zwischendampfstößen zu erreichender Druck: $\geq 1380$ mbar

\section{Desinfektion}

Dampftemperatur: $109^{\circ} \mathrm{C}$

Einwirkungszeit: $27 \mathrm{~min}$

Wirkungsbereich: ABC

\section{Geprüfter und anerkannter Apparatetyp:} KEA 100

\subsection{System \\ Schlumbohm-CISA}

Die bei der Eintragung vom Robert KochInstitut erteilten Auflagen, insbesondere bezüglich der Art des Abfalls und seiner Verpackung, sind zu beachten.

\section{Betriebsdaten}

a) Programm für feste Abfälle Luftentfernung Anzahl der Evakuierungsphasen: 5 In den Evakuierungsphasen zur erreichender Druck: $\leq 150$ mbar Bei den Zwischendampfstößen zu erreichender Druck: $\geq 1800$ mbar

\section{Desinfektion \\ Dampftemperatur: $134^{\circ} \mathrm{C}$ \\ Einwirkungszeit: $15 \mathrm{~min}$ \\ Wirkungsbereich ABC}

b) Programm für flüssige Abfälle Luftentfernung Anzahl der Evakuierungsphasen: 1 In der Evakuierungsphase zur erreichender Druck: $\leq 100$ mbar

\section{Desinfektion}

Dampftemperatur: $121^{\circ} \mathrm{C}$

Einwirkungszeit: $20 \mathrm{~min}$

Wirkungsbereich ABC
Geprüfte und anerkannte Apparatetypen: CISA 6464, CISA 6410, CISA 6412

\subsection{System Webeco}

Die bei der Eintragung vom Robert KochInstitut erteilten Auflagen, insbesondere bezüglich der Art des Abfalls und seiner Verpackung, sind zu beachten.

\section{Betriebsdaten}

a) $105^{\circ} \mathrm{C}$ - Programm

Luftentfernung

Anzahl der Evakuierungsphasen: 7

In den Evakuierungsphasen zu erreichender Druck:

1. Phase: $\leq 90$ mbar

Folgende Phasen: $\leq 200$ mbar

Bei den Zwischendampfstößen zu erreichender Druck: $\geq 1250$ mbar

Der Druck wird bei dem 1. Zwischendampfstoß $60 \mathrm{~s}$ und bei den weiteren Dampfstößen jeweils 30 s gehalten.

\section{Desinfektion}

Dampftemperatur: $105^{\circ} \mathrm{C}$

Einwirkungszeit: $30 \mathrm{~min}$

Wirkungsbereich: ABC

Geprüfte und anerkannte Apparatetypen: EMD 217, EMD 224, EMD 230, EMD 235, EMD 250, E 14-Labor, E 16-Labor, E 18-Labor, E 24-Labor, E 26-Labor, E 28-Labor, EST-Labor 110, EST-Labor 115, EST-Labor 120, EST-Labor 210, EST-Labor 215, EST-Labor 220

b) Programm Feststoffe Luftentfernung Anzahl der Evakuierungsphasen: 2 In den Evakuierungsphasen zu erreichender Druck:

1. Phase: $\leq 120$ mbar

2. Phase: $\leq 200$ mbar

Bei dem Zwischendampfstoß zu erreichender Druck: $\geq 1900$ mbar

\section{Desinfektion}

Dampftemperatur: $134^{\circ} \mathrm{C}$

Einwirkungszeit: $5 \mathrm{~min}$

Wirkungsbereich: ABC

Geprüfte und anerkannte Apparatetypen:

CS/VF, CS/VFT, CS/VFKT c) Programm Flüssigkeiten

Luftentfernung

Anzahl der Evakuierungsphasen: 1

In der Evakuierungsphase zu erreichender Druck: $\leq 120$ mbar

Desinfektion

Dampftemperatur: $115^{\circ} \mathrm{C}$

Einwirkungszeit (nach Erreichen von $115^{\circ} \mathrm{C}$ an einem Temperaturfühler in dem Gut bzw. in einem Referenzgefäß): $10 \mathrm{~min}$ Wirkungsbereich: ABC

Geprüfte und anerkannte Apparatetypen: CS/VFT, CS/VFKT, E 14-Labor, E 16-Labor, E 18-Labor, E 24-Labor, E 26-Labor, E 28-Labor, EST-Labor 110, EST-Labor 115, EST-Labor 120, EST-Labor 210, ESTLabor 215, EST-Labor 220

d) Programm Petrischalen

Das Gesamtvolumen der eingebrachten Nährmedien darf 21 nicht überschreiten.

Luftentfernung

Anzahl der Evakuierungsphasen: 1

In der Evakuierungsphase zu erreichender Druck: $\leq 120$ mbar

Desinfektion

Dampftemperatur: $121^{\circ} \mathrm{C}$

Einwirkungszeit (nach Erreichen von $121^{\circ} \mathrm{C}$ an einem freiliegenden Temperaturfühler): $10 \mathrm{~min}$

Wirkungsbereich: ABC

Geprüfte und anerkannte Apparatetypen: CS/VF, CS/VFT, CS/VFKT

e) $134^{\circ} \mathrm{C}-$ Programm

Luftentfernung

Anzahl der Evakuierungsphasen: 7

In den Evakuierungsphasen zu erreichender Druck:

1. Phase: $\leq 90$ mbar

Folgende Phasen: $\leq 200$ mbar

Bei den Zwischendampfstößen zu erreichender Druck: $\geq 1900$ mbar

Der Druck wird bei dem 1. Zwischendampfstoß $60 \mathrm{~s}$ und bei den weiteren Dampfstößen jeweils 30 s gehalten.
Desinfektion
Dampftemperatur: $134^{\circ} \mathrm{C}$
Einwirkungszeit: $10 \mathrm{~min}$
Wirkungsbereich: ABC 
Geprüfte und anerkannte Apparatetypen: E 14-Labor, E 16-Labor, E 18-Labor, E 24-Labor, E 26-Labor, E 28-Labor, ESTLabor 110, EST-Labor 115, EST-Labor 120, EST-Labor 210, EST-Labor 215, EST-Labor 220

f) Programm: P 1 Abfalldesinfektion, Feststoffe; Müllsterilisation $134^{\circ} \mathrm{C}$ Luftentfernung

Vor der ersten Evakuierungsphase wird Dampf in die Kammer bis zu einem Druck von $\geq 3000$ mbar eingegeben.

Dieser Druck wird 5 min gehalten.

Anzahl der Evakuierungsphasen: 4

In den Evakuierungsphasen zu erreichender Druck: $\leq 200$ mbar

Bei den Zwischendampfstößen zu erreichender Druck: $\geq 1900$ mbar mit jeweils 30 s Haltezeit

\section{Desinfektion}

Dampftemperatur: $134^{\circ} \mathrm{C}$

Einwirkungszeit: $20 \mathrm{~min}$

Wirkungsbereich: ABC

Geprüfte und anerkannte Apparatetypen: WEBECO: EC 140/EC 240-Lab, EC 160/ EC 260-Lab bzw.

Matachana: S-1004 I-E1/-E2,

S-1006 I-E1/-E2

g) Programm: P 2 Flüssigkeiten, Abfall, offene Flüssigkeiten 1 L3

Luftentfernung

Anzahl der Evakuierungsphasen: 1

In der Evakuierungsphase zu erreichender Druck: $\leq 600$ mbar

\section{Desinfektion}

Dampftemperatur: $121^{\circ} \mathrm{C}$

Einwirkungszeit: $20 \mathrm{~min}$

Wirkungsbereich: ABC

Geprüfte und anerkannte Apparatetypen: WEBECO: EC 140/EC 240-Lab, EC 160/ EC 260-Lab bzw.

Matachana: S-1004 I-E1/-E2,

S-1006 I-E1/-E2

\subsubsection{Spezielle Verfahren}

\subsection{System Göldner I}

Das Verfahren ist gekennzeichnet durch:

1. Zerkleinern des Gutes in einem ge schlossenen System
2. Aufheizen des Gutes in einer Förder schnecke (Ölmanteltemperatur $115^{\circ} \mathrm{C}$ )

3. Desinfektion des Gutes durch Sattdampf in einer Temperaturhalteschnecke (Ölmanteltemperatur $115^{\circ} \mathrm{C}$ )

Die bei der Eintragung vom Robert Koch-Institut erteilten Auflagen, insbesondere bezüglich der Art des Abfalls und seiner Verpackung sowie bezüglich der Maßnahmen bei Betriebsende und Betriebsstörungen, sind zu beachten.

\section{Betriebsdaten}

Desinfektionstemperatur: $110^{\circ} \mathrm{C}$

Einwirkungszeit: mindestens $45 \mathrm{~min}$ (definiert über die Geschwindigkeit der Temperaturhalteschnecke: $0,7 \mathrm{U} / \mathrm{min}$ )

Wirkungsbereich: ABC

\section{Geprüfter und anerkannter Apparatetyp:} LOGMED

\subsection{System Göldner II}

Das Verfahren ist gekennzeichnet durch:

1. Befüllen der Anlage und Zerkleinern des Gutes in einem geschlossenen System, Dauer 10 min, KorngröBe $20 \times 30 \mathrm{~mm}$

2. Zugabe von 51 Wasser in die Expositionsschnecke

3. Aufheizen des zerkleinerten Gutes in der Expositionsschnecke (Ölmanteltemperatur $115^{\circ} \mathrm{C}$ ) mittels Sattdampf auf $98^{\circ} \mathrm{C}$, halten dieser Temperatur für $6 \mathrm{~min}$, aufheizen auf $116^{\circ} \mathrm{C}$

4. Desinfektion des Gutes durch Sattdampf in der Expositionsschnecke (Ölmanteltemperatur $115^{\circ} \mathrm{C}$ )

5. Desinfektion des Kondensats und der sich aus dem zerkleinerten Abfall angesammelten Flüssigkeit in einem separaten Desinfektionsdruckbehälter

Die bei der Eintragung vom Robert KochInstitut erteilten Auflagen, insbesondere bezüglich der Maßnahmen bei Betriebsende und Betriebsstörungen, sind zu beachten.

\section{Betriebsdaten}

Expositionsschnecke

Desinfektionstemperatur: $115^{\circ} \mathrm{C}$
Einwirkungszeit: 15 min (Halten der Temperatur im Temperaturband durch Regelung über 3 in der Expositionsschnecke verteilte und miteinander verbundene Temperaturfühler im Abgleich mit der theoretischen Sattdampfkurve)

Wirkungsbereich: ABC

Desinfektionsdruckbehälter (Flüssigkeitsund Kondensatbehandlung):

Desinfektionstemperatur: $115^{\circ} \mathrm{C}$

Einwirkungszeit: $15 \mathrm{~min}$

Wirkungsbereich: ABC

Geprüfter und anerkannter Apparatetyp: LOGMED II

\subsection{System Sterifant}

Das Verfahren ist gekennzeichnet durch:

1. Zugabe von Wasser zum Desinfektionsgut (insgesamt 21 pro Desinfektionsbehälter)

2. Mehrfaches Evakuieren der Desinfektionsbehälter im Wechsel mit Dampfeinströmung, die Dauer der Dampfeinströmungsphase ist durch die Leistung des Dampfgenerators (9 KW) festgeschrieben

3. Aufheizen des Desinfektionsgutes mittels Mikrowellen

4. Desinfektion des Gutes unter Sattdampfbedingungen

Die bei der Eintragung vom Robert KochInstitut erteilten Auflagen, insbesondere bezüglich der Art des Abfalls und seiner Verpackung, sind zu beachten.

\section{Betriebsdaten}

Luftentfernung

Anzahl der Evakuierungsphasen: 5

In den Evakuierungsphasen zu erreichender Druck:

1. bis 4 . Phase: $\leq 500$ mbar

5. Phase: $\leq 630$ mbar

in den Zwischendampfstößen zu erreichender Druck: $\geq 1430$ mbar. Dieser Druck wird jeweils 5 min gehalten.

\section{Desinfektion}

Dampftemperatur: $105^{\circ} \mathrm{C}$

Einwirkungszeit: $20 \mathrm{~min}$

Wirkungsbereich: ABC 
Geprüfter und anerkannter Apparatetyp: STERIFANT 90/4

\subsubsection{Sonderverfahren}

\subsubsection{System Meteka - Sonderverfahren für flüssige Abfälle}

Das Verfahren ist gekennzeichnet durch: Erhitzung der flüssigen Abfälle in speziellen Behältern mittels Mikrowellen.

Die bei der Eintragung vom Robert Koch-Institut erteilten Auflagen, insbesondere bezüglich der Art des Abfalls und seiner Verpackung, sind zu beachten.

\section{Betriebsdaten}

Desinfektion

Desinfektionstemperatur: $100^{\circ} \mathrm{C}$

Einwirkungszeit: $25 \mathrm{~min}$

Wirkungsbereich: ABC

Geprüfter und anerkannter Apparatetyp: MEDISTER 60 Liquid

\subsubsection{System Meteka -} Sonderverfahren für Nassabfälle I

Das Verfahren ist gekennzeichnet durch: Erhitzung der Abfälle nach Wasserzugabe in speziellen Behältern mittels Mikrowellen.

Die bei der Eintragung vom Robert Koch-Institut erteilten Auflagen, insbesondere bezüglich der Art des Abfalls und seiner Verpackung, sind zu beachten. Das Verfahren ist nur geeignet für Abfälle, die ausreichend Wasser enthalten (mikrobiologische Kulturen, Blutproben, Stuhlproben, Drainagebeutel bzw. -flaschen, Blutbeutel).

\section{Betriebsdaten}

Desinfektion

Desinfektionstemperatur: $100^{\circ} \mathrm{C}$

Einwirkungszeit: $25 \mathrm{~min}$

Wirkungsbereich: ABC

Geprüfte und anerkannte Apparatetypen: MEDISTER 10, MEDISTER 60, MEDISTER 160

\subsubsection{System Meteka - Sonderverfahren für Nassabfälle II}

Das Verfahren ist nur geeignet für Abfälle, die ausreichend Wasser enthalten (mikrobiologische Kulturen, Blutproben, Stuhlproben, Drainagebeutel bzw. -flaschen, Blutbeutel, Dialysesysteme).

Das Verfahren ist gekennzeichnet durch die Erhitzung der Abfälle nach Wasserzugabe in speziellen Behältern mittels Hochfrequenztechnik (Mikrowellenbereich).

\section{Betriebsdaten}

Desinfektionstemperatur: $121^{\circ} \mathrm{C}$

Einwirkungszeit: $20 \mathrm{~min}$

Wirkungsbereich: ABC

Geprüfter und anerkannter Apparatetyp: MEDISTER 360

\subsection{Sonderverfahren zur Behandlung von HEPA-Filtern in Sicherheitswerkbänken (Klasse 2)}

Verfahren zur Desinfektion von Filtern in Sicherheitswerkbänken durch Begasung müssen speziell für diese Anwendung geprüft sein. Dies schließt den Nachweis der Wirksamkeit des Verfahrens bei dem jeweiligen Typ der Sicherheitswerkbank und dem Typ des verwendeten Filters für den erforderlichen Wirkungsbereich ein.

\subsubsection{System STERIS (VHP $\mathrm{H}_{2} \mathrm{O}_{2}$-Verfahren)}

Das Verfahren ist gekennzeichnet durch:

1. Entzug der Luftfeuchtigkeit

2. Verdampfen von Wasserstoffperoxid (35\%ig Vaprox ${ }^{\circledR}$ ) und Durchströmen der HEPA-Filter von Sicherheitswerkbänken mit Wasserstoffperoxid

3. Durchströmen der HEPA-Filter der Sicherheitswerkbank bei laufendem Ventilator und weiterer Verdampfung von Wasserstoffperoxid

\section{Betriebsdaten}

1. Entfeuchtung

Luftstrom: $32 \mathrm{~m}^{3} / \mathrm{h}$

Zeit: $10 \mathrm{~min}$

Temperaturbereich: $16-37^{\circ} \mathrm{C}$

zu erreichende Luftfeuchte: $\leq 5 \mathrm{mg} / \mathrm{l}$
2. Konditionierung Luftstrom: $18 \mathrm{~m}^{3} / \mathrm{h}$

Zeit: $10 \mathrm{~min}$

Dosierung von Vaprox: 3,7 g/min

Temperaturbereich: $16-37^{\circ} \mathrm{C}$ $\mathrm{zu}$ erreichende $\mathrm{H}_{2} \mathrm{O}_{2}$-Konzentration am Ende der Konditionierung: 1,3 mg/l

3. Desinfektion

Luftstrom: $18 \mathrm{~m}^{3} / \mathrm{h}$

Einwirkzeit: $200 \mathrm{~min}$

Dosierung von Vaprox: $3,5 \mathrm{~g} / \mathrm{min}$

Temperaturbereich: $16-37^{\circ} \mathrm{C}$

$\mathrm{H}_{2} \mathrm{O}_{2}$-Konzentration während der Desinfektionsphase: $1,1 \mathrm{mg} / \mathrm{l}$

4. Belüftung Luftstrom: $33 \mathrm{~m}^{3} / \mathrm{h}$

Zeit: $90 \mathrm{~min}$

Temperaturbereich: $16-37^{\circ} \mathrm{C}$ $\mathrm{zu}$ erreichende $\mathrm{H}_{2} \mathrm{O}_{2}$-Konzentration am Ende der Belüftung: $\leq 1 \mathrm{ppm}$ Wirkungsbereich: ABCD

Geprüfte und anerkannte Apparatetypen: VHP1001G, VHP1000ED-S

Geprüfter Filtertyp:

HEPA-Filter (Glasfaser und Neopren) in Sicherheitswerkbänken der Klasse 2

Die bei der Eintragung vom Robert KochInstitut erteilten Auflagen, insbesondere bezüglich der Art der HEPA-Filter und des Innenvolumens der Werkbänke, sind zu beachten. Diese sind vor der Anwendung des Verfahrens beim Hersteller zu erfragen.

Hersteller bzw. Lieferfirma: STERIS Deutschland GmbH

\subsubsection{Begasung mit Formaldehyd}

Das Verfahren ist gekennzeichnet durch:

1. Verdampfen von Formaldehyd (18\%ig) und Durchströmen der HEPA-Filter der Sicherheitswerkbank mit Formaldehyd bei laufendem Ventilator bei hoher Luftfeuchtigkeit und Temperaturen $\geq 70^{\circ} \mathrm{C}$

2. Verdampfen von Ammoniak (25\%ig) und Durchströmen der HEPA-Filter der Sicherheitswerkbank mit Ammoniak bei laufendem Ventilator 


\section{Betriebsdaten}

1. Vorbereitung

- Einhausen der Werkbank mit gleichzeitiger Wärmeisolierung

- Erhöhung der Luftfeuchtigkeit und Temperatur durch Wasserbäder und Wärmestrahler im Arbeitsraum

- zu erreichende Luftfeuchte: $100 \%$ rel. Luftfeuchte (Messung hinter dem Abluftfilter)

- zu erreichende Temperatur: $\geq 70^{\circ} \mathrm{C}$ (Messung hinter dem Abluftfilter)

- Luftstrom: Umluftbetrieb (reduzierte Gebläseleistung/Stand-by-Betrieb)

2. Desinfektion

- Verdampfung von $1000 \mathrm{ml}$ 18\%iger Formaldehydlösung

- Luftstrom: Umluftbetrieb (reduzierte Gebläseleistung/Stand-by-Betrieb)

- zu erreichende Luftfeuchte: $\sim 100 \%$ rel. Luftfeuchte (Messung hinter dem Abluftfilter)

- Temperatur: $\geq 70^{\circ} \mathrm{C}$ (Messung hinter dem Abluftfilter)

- Einwirkzeit: $9 \mathrm{~h}$

3. Neutralisation

- Verdampfung von $300 \mathrm{ml}$ 25\%iger Ammoniaklösung

- Luftstrom: Umluftbetrieb (reduzierte Gebläseleistung/Stand-by-Betrieb)

- Temperatur: Raumtemperatur

- Einwirkzeit: $1 \mathrm{~h}$

- Belüftung bis zum Erreichen des MAK-Wertes: Formaldehyd-Konzentration $\leq 0,3 \mathrm{ppm}$

Wirkungsbereich: ABCD

Geprüfter Filtertyp:

HEPA-Filter (Glasfaser/Neopren) in mikrobiologischen Sicherheitswerkbänken der Klasse 2

Für die ordnungsgemäße Durchführung der Desinfektion sind nachfolgende Auflagen zu beachten:

- Dieses Desinfektionsverfahren ist nur von qualifiziertem Personal, das den Befähigungsschein nach TRGS 522 besitzt, durchzuführen.

- Das Verfahren kann nur für die oben angegebenen Filter in Sicherheitswerkbänken mit einer Länge der
Arbeitsöffnung von 1,2 m angewendet werden. Für andere Filter oder Sicherheitswerkbänke mit anderen $\mathrm{Ab}$ messungen ist eine erneute Validierung mit Bioindikatoren - Geobacillus stearothermophilus (KBE 106 ) nach DIN EN ISO 11138-5 - im Abluftfilter erforderlich.

- Die Desinfektion von Filtern in Sicherheitswerkbänken muss mit geeigneter persönlicher Schutzausrüstung durchgeführt werden. Die Anforderungen des Arbeitsschutzes (z. B. TRGS 522, GefahrstoffVO) sind einzuhalten.

- Die Nachbehandlung der Filter unterliegt den jeweiligen landesrechtlichen Vorschriften zur Abfallentsorgung.

\section{Anschriften der Hersteller bzw. Lieferfirmen:}

\author{
Anti-Germ Deutschland GmbH \\ 87700 Memmingen \\ http://www.anti-germ.de
}

\author{
Antiseptica \\ Chem. pharm. Produkte GmbH \\ 50259 Pulheim \\ http://www.antiseptica.com
}

van Baerle Chem. Fabrik GmbH \& Co. 64575 Gernsheim/Rhein

http://www.van-baerle.com

\author{
Becker Chemie GmbH \\ 33818 Leopoldshöhe \\ http://www. becker-chemie.de \\ Belimed AG \\ CH-6275 Ballwil \\ http://www.belimed.com \\ Belimed Deutschland $\mathrm{GmbH}$ \\ 84453 Mühldorf \\ http://www.belimed.com
}

\section{Belimed Sauter}

vertreten durch Belimed Deutschland $\mathrm{GmbH}$

BHT Hygiene Technik GmbH 86368 Gersthofen

http://www.bht-hygienetechnik.de

Bode Chemie GmbH

22507 Hamburg

http://www.bode-chemie.de
B. Braun Melsungen AG
34209 Melsungen
http://www.bbraun.de

BÜFA Reinigungssysteme GmbH \& Co KG 26015 Oldenburg

http://www.buefa.de

BurnusHychem $\mathrm{GmbH}$

36396 Steinau a. d. Straße

http://www.burnushychem.com

\author{
Christeyns $\mathrm{GmbH}$ \\ 77654 Offenburg \\ http://www.christeyns.com
}




\author{
CHT Beitlich $\mathrm{GmbH}$ \\ 72002 Tübingen \\ http://www.cht.com \\ CMB Maschinenbau und Handels $\mathrm{GmbH}$ \\ A-8051 Graz \\ http://www.christof-group.at

\section{Desomed \\ Dr. Trippen GmbH \\ 79020 Freiburg \\ http://www.desomed.de}

Diversey

Deutschland $\mathrm{GmbH} \& \mathrm{Co}$. OHG

68219 Mannheim

http://www.diversey.com

System Drauschke

GÖK Consulting AG

10587 Berlin

http://www.goek-consulting.de

\section{Dreiturm $\mathrm{GmbH}$}

36392 Steinau a. d. Straße

http://www.dreiturm.de

\section{Ecolab $\mathrm{GmbH}$}

40766 Monheim

http://www.ecolab.com

Enzian Seifen

72542 Metzingen

http://www.enzian-seifen.de

ERVE Deutschland

59513 Lüdenscheid

http://www.erveschuster.de

Fink Tec GmbH

59069 Hamm

http://www.finktec.com

Göldner Umwelt-\& Hygienetechnik GmbH 06188 Landsberg

http://www.logmed.de

Hagleitner Hygiene International $\mathrm{GmbH}$ A-5700 Zell am See

http://www. hagleitner.com

\section{HIMED GmbH}

48163 Münster

http://www.himed.de
Holzner Medizintechnik GmbH

69226 Nußloch

http://www.holzner.net

HP Medizintechnik $\mathrm{GmbH}$

85764 Oberschleißheim

http://www.hp-med.com

igefa Handelsgesellschaft mbH \& Co. KG

16356 Ahrensfelde

http://www.igefa.de

Jensen $\mathrm{GmbH}$

31175 Harsum

http://www.jensen-group.com

Kaniedenta GmbH \& Co KG

32052 Herford

http://www.kaniedenta.de

Kesla Pharma Wolfen GmbH

06803 Greppin

http://www.kesla.de

Johannes Kiehl KG

85235 Odelzhausen

http://www. kiehl-group.com

Kleen Purgatis GmbH

32120 Hiddenhausen

http://www.kleen-purgatis.de

GUSTAV KOCH GmbH \&Co. KG

50169 Kerpen

http://www.gustav-koch.de

Chemische Fabrik Kreussler \& Co. GmbH 65082 Wiesbaden

http://www.kreussler.com

Laboratorium Dr. H.D. Deppe

47906 Kempen

http://www.dr-deppe.de

Lancer S.A. Industrie

F-31170 Tournefeuille

http://www.lancer.fr

F. \& M. Lautenschläger GmbH \& Co. KG

50972 Köln

http://www. Lautenschlaeger.net

Lysoform

Dr. Hans Rosemann GmbH

12247 Berlin

http://www.lysoform.de
Merz Hygiene $\mathrm{GmbH}$

60048 Frankfurt a. M.

http://www.merz.de

Meteka $\mathrm{GmbH}$

A-8750 Judenburg

http://www.meteka.com

Fritz Osk. Michallik

75403 Mühlacker

http://www.michalik.com

Miele \& Cie. GmbH \& Co.

33325 Gütersloh

http://www.miele-professional.de

$M M M$

Münchner Medizin Mechanik GmbH

82141 Planegg

http://www.mmmgroup.com

mobiloclean Handelsgruppe GmbH \& Co.

KG

80935 München

http://www.mobiloclean.com

Mundipharma $\mathrm{GmbH}$

65533 Limburg (Lahn)

http://www.mundipharma.de

NORDENTA Handelsgesellschaft $\mathrm{mbH}$

22012 Hamburg

http://www.nordenta.de

Nova Praxis-Hygiene oHG

82256 Fürstenfeldbruck

http://www.nova-praxis-hygiene.de

Dr. Nüsken Chemie GmbH

59158 Kamen

http://www.drnuesken.de

orochemie

70798 Kornwestheim

http://www.orochemie.de

POC Peter Ott Consulting

66832 Schmelz

Riebesam GmbH \& Co. KG

39307 Genthin

http://www.riebesam.de

R. Ringeisen

73033 Göppingen

http://www.steri-ring.de 


\section{Rösch Germany GmbH \\ 88131 Lindau \\ http://www.roesch-waschmittel.de

Sanitätsamt der Bundeswehr
80637 München
SARAYA Europe
B-1020 Brüssel
http://www.saraya-europe.com

Schlumbohm GmbH

21079 Hamburg

http://www.schlumbohm-medizintech-

nik.de

\section{Dr. Schnell Chemie GmbH \\ 80807 München \\ http://www.dr-schnell.de}

Schülke \& Mayr GmbH

22840 Norderstedt

http://www.schuelke.com

Dr. Schumacher $\mathrm{GmbH}$

34201 Melsungen

http://www.schumacher-online.com

SCHUSTER-Chemie GmbH \& Co. KG

88131 Lindau

http://www.erveschuster.de

\section{Seeger $\mathrm{GmbH}$}

72309 Balingen

http://www.seeger-sauber.de

\author{
Seitz GmbH \\ 65825 Kriftel \\ http://www.seitz24.com

Steris Deutschland GmbH
50933 Köln
http://www.steris.com

Sterifant International Holding AG

L-1466 Luxembourg

http://www.sterifant-gmbh.com

Tana PROFESSIONAL Werner \& Mertz $\mathrm{GmbH}$

55033 Mainz

http://www.tana.de

Textil Catering Service GmbH

CH-9244 Niederuzwil
Wachendorff-Chemie GmbH

53831 Troisdorf

http://www.rheosol.de

Webeco GmbH \& Co. KG

23603 Bad Schwartau

http://www.webeco.de

Chemische Fabrik Dr. Weigert Beteiligungsgesellschaft $\mathrm{mbH}$

20530 Hamburg

http://www.drweigert.de

August Wencke OHG

28701 Bremen

http://www.august-wencke.de

Ernst Zeiss Wäschereibedarf Vertriebs $\mathrm{GmbH}$

64293 Darmstadt

\section{Anhang zur Liste der vom \\ Robert Koch-Institut geprüften und anerkannten Desinfektionsmittel und-verfahren}

Desinfektion von speziellen Oberflächen Die im Abschn. 2.2 der RKI-Liste aufgeführten Mittel und Verfahren beziehen sich auf eine Anwendung auf festen wischdesinfizierbaren Oberflächen im medizinischen Bereich.

Im Hinblick auf eine nachgewiesene Wirksamkeit ergibt sich auch in anderen Zusammenhängen, z. B. zur Abwendung von Gefahren durch humanpathogene Erreger einschließlich bakterieller Sporenbildner auf anderen Oberflächen, wie z. B. der persönlichen Schutzausrüstung von Rettungskräften, der Wunsch nach verlässlichen Hinweisen zur sachgerechten Anwendung geeigneter Desinfektionsmittel.

Für den speziellen Fall der Anwendung auf Oberflächen von PSA wurden im Rahmen eines Forschungsprojektes Prüfmethoden entwickelt, die den Besonderheiten dieser Anwendung Rechnung tragen [s. Lemmer et al. 2012 (14)]. Um dem Bedürfnis nach Information zu diesem Thema an zentraler Stelle gerecht zu werden, werden in diesem Anhang zur Liste grundlegende Ergebnisse aus diesen Untersuchungen (Konzentration/ZeitRelation und Volumina) für ausgewählte Wirkstoffe oder Desinfektionsmittel aufgeführt.

Es wird ausdrücklich darauf hingewiesen, dass bei der konkreten Anwendung in dem hier beschriebenen Kontext die spezifischen Umstände des Einsatzes berücksichtigt werden müssen. Hierzu dient eine entsprechende Validierung unter den jeweiligen Praxisbedingungen [s. Lemmer et al. 2012 (14)], welche nicht Gegenstand der Listung ist.

Auf die PSA-Richtlinie und die TRBA 130 und 250 wird ausdrücklich hingewiesen.

Desinfektion hydrophober, (flexibler) Oberflächen

Konzentration: 5\% Wofasteril E400 und 0,5\% Alkapur N

Einwirkzeit: $5 \mathrm{~min}$

Anwendungsmenge: $\geq 50 \mathrm{ml} / \mathrm{m}^{2}$

Wirkungsbereich: ABC 